\title{
Statistics of magnetic reconnection in two-dimensional magnetohydrodynamic turbulence
}

\author{
S. Servidio, ${ }^{1,2}$ W. H. Matthaeus, ${ }^{1}$ M. A. Shay, ${ }^{1}$ P. Dmitruk, ${ }^{3}$ P. A. Cassak,${ }^{4}$ and M. Wan ${ }^{1}$ \\ ${ }^{1}$ Department of Physics and Astronomy and Bartol Research Institute, University of Delaware, \\ Newark, Delaware 19716, USA \\ ${ }^{2}$ Dipartimento di Fisica, Università della Calabria, Cosenza I-87036, Italy \\ ${ }^{3}$ Departamento de Física, Facultad de Ciencias Exactas y Naturales, Universidad de Buenos Aires, \\ Ciudad Universitaria, Buenos Aires 1428, Argentina \\ ${ }^{4}$ Department of Physics, West Virginia University, Morgantown, West Virginia 26506, USA
}

(Received 3 December 2009; accepted 1 March 2010; published online 31 March 2010)

\begin{abstract}
The nonlinear dynamics of magnetic reconnection in turbulence is investigated through direct numerical simulations of decaying, incompressible, two-dimensional magnetohydrodynamics. Recently, it was shown by Servidio et al. [Phys. Rev. Lett. 102, 115003 (2009)] that in fully developed turbulence complex processes of reconnection occur locally. Here, the main statistical features of these multiscale reconnection events are further described, providing details on the methodology. It is found that is possible to describe the reconnection process in turbulence as a generalized local Sweet-Parker process in which the parameters are locally controlled by the turbulence cascade, thus providing a step toward reconciling classical turbulence analysis with reconnection theory. This general description of reconnection may be useful for laboratory and space plasmas, where the presence of turbulence plays a crucial role. (C) 2010 American Institute of Physics. [doi:10.1063/1.3368798]
\end{abstract}

\section{INTRODUCTION}

Magnetic reconnection is a nonlinear process that occurs in many space, astrophysical, and laboratory systems, ${ }^{1-3}$ such as near the sun's surface, ${ }^{4}$ in the magnetosphere, ${ }^{5,6}$ in the solar wind, ${ }^{7}$ in the strongly turbulent magnetosheath, ${ }^{8,9}$ and in laboratory devices. ${ }^{10-14}$ The underlying common feature for these systems is the presence of an inhomogeneous magnetic field that changes rapidly across a very narrow region. Generally a strong peak in the electric current density is present. Reconnection implies the presence of a magnetic $\mathrm{X}$-type neutral point ${ }^{15}$ in two dimensions, and more generally a change in magnetic topology ${ }^{6,16}$ resulting in the conversion of magnetic into kinetic energy. ${ }^{17,18}$ The result is the formation of a new magnetic island associated with a characteristic out-flow pattern.

Theoretical efforts uncovered much of the basic physics of reconnection, especially in idealized geometries. Sweet ${ }^{17}$ and Parker ${ }^{18}$ employed conservation of mass, pressure balance, and continuity of the electric field to reveal the essential large scale dynamics of magnetic reconnection in the framework of two-dimensional (2D) magnetohydrodynamics (MHD). In this simple configuration, a neutral sheet separating plasma regions contains oppositely directed magnetic fields. In a small region called the "diffusion region" near a magnetic X-type null point, there is lower magnetic pressure and a higher plasma pressure. This produces a plasma outflow along the neutral sheet, and accordingly flow into the neutral sheet from the strong field regions. The flow carries magnetic flux into the diffusion region where the field lines change topology. The Sweet-Parker model operates at a finite value of resistivity, but the rate of reconnection, measured through the in-flow speed (usually normalized to the
Alfvénic speed), decreases with increasing magnetic Reynolds number. Driving ${ }^{19}$ and kinetic effects ${ }^{20}$ can have a strong influence on reconnection.

Since it might occur in any region separating topologically distinct magnetic flux structures, reconnection might be expected to be of importance in more general circumstances, including magnetohydrodynamic (MHD) turbulence. ${ }^{21}$ For example, it is difficult to envision a turbulent cascade that proceeds without change in magnetic topology. Furthermore, turbulence may provide a kind of unbiased or natural local boundary condition that provides a view of reconnection relatively unaffected by arbitrary (imposed) conditions. Although some suggestions have been made regarding both the general role of reconnection in MHD turbulence ${ }^{22-25}$ and the impact of small scale turbulence on reconnection of large structures, ${ }^{21,22,26-28}$ until recently no quantitative account has been given of reconnection that occurs as an integral part of the turbulence cascade. ${ }^{29}$

In the past decades, numerical simulations played a very important role in the investigation of the dynamics of reconnection. Most early numerical experiments addressed the study of the tearing instability and its saturation. ${ }^{30,31}$ The forced, steady state reconnection envisioned by Petschek, ${ }^{32}$ which incorporates an externally supported velocity field, and admits more complex and faster reconnection than Sweet-Parker, has been extensively studied as well. ${ }^{19}$ Most of these models are essentially in 2D, and assume simplified geometries and controlled ambient conditions, with the plasma on either side of the dissipation region having identical magnetic field strengths, and with well-defined, specified boundary conditions. The method of initializing reconnection is important and varied widely in simulation studies. With very low amplitude perturbations, linear tearing insta- 
bilities characterize the evolution, until saturation of some kind is attained. The reconnection process can also be initiated by pressure ${ }^{32}$ or electromagnetic stresses at the boundary (e.g., Refs. 33 and 34). When an initial perturbation is large enough, a fully nonlinear state can be rapidly achieved (e.g., Ref. 20).

Reconnection may be self-sustaining if boundary conditions permit, or it may continue until available magnetic flux is exhausted. In many cases the reconnecting system has been idealized as occurring in a limited spatial region defined by the simulation "box." The magnetic field is often arbitrarily chosen to be straight at the inflow-side boundaries and simplified "outflow" boundaries are employed. However a complementary perspective is that such simplified or idealized conditions are not easily realized in nature: naturally occurring plasmas may frequently be found a fully nonlinear turbulent state. In these conditions, the dynamical processes associated with reconnection are likely to occur in a less controlled environment, one in which the conditions are not set in advance or in a controlled way. Instead the externally controlled conditions to which a reconnection zone responds are themselves set in a dynamic way as a consequence of the global nonlinear dynamics of the system. ${ }^{25}$ It is this scenario of reconnection as an intrinsic feature of turbulence that we address here.

There have been a variety of previous studies that examined the effect on the reconnection process of finite amplitude broadband fluctuations (turbulence), The first studies of this type, ${ }^{21,35}$ in periodic geometry, demonstrated that topology change and dynamical activity centered around magnetic $\mathrm{X}$-points are integral features of turbulent relaxation. Furthermore the presence of fluctuations (whatever their origin) in the reconnection zone induces multiple X-points, the appearance of small secondary islands and an acceleration of the reconnection process. ${ }^{36}$ Detailed examination of spectra, the spatial structure of the fluctuation field, and various correlations in turbulent reconnection ${ }^{22}$ developed more detail in the emerging picture of the close relationship between properties of reconnection and properties of MHD turbulence. Later, quantitative study showed that ${ }^{37}$ reconnection rates in 2D periodic geometry are increased systematically by finite amplitude fluctuations at early times, leading to a saturated rate later in the turbulence development. Later studies further examined this scenario, by computing effects of turbulence on the effective resistivity, introducing threedimensional effects, and further describing the feedback associated with the turbulence. . $^{27,28,38}$

Recent studies have adopting varying approaches that confirm the key role of turbulence and fluctuations on reconnection. In one recent paper ${ }^{39}$ three-dimensional turbulent fluctuations are directly driven in the reconnection layer, emulating the effects of a strongly turbulent medium. Another related study ${ }^{40}$ carried out driven two-dimensional computations, verifying that turbulence enhances reconnection rates to order-one Alfvénic rates at high mechanical and magnetic Reynolds numbers.

Other studies revived interest in the direct effects of the presence of small scale fluctuations within the reconnection layer, originally envisioned mainly due to pre-existing fluc- tuations, but possibly augmented by instability near the current sheet. ${ }^{36}$ The recent examination of the direct effect of complexity in the reconnection layer employed kinetic simulation $^{41}$ as well as MHD simulation ${ }^{42,43}$ to show that thin laminar current sheets may be subjected to growth of secondary islands. Once present these then can accelerate reconnection as described earlier. ${ }^{36}$

However, even with the substantial progress that has been made, a full picture of the relationship between reconnection and turbulence is yet to emerge. In particular, almost all previous studies focused on the influence of turbulence on large scale reconnecting magnetic fields. The problem of reconnection that may occur between numerous adjoining turbulent structures ${ }^{35}$ received relatively less attention. However a better understanding of reconnection within a fully turbulent scenario may be significant in the areas of space and astrophysical plasmas. In this regard, the present paper extends an approach that seems particularly timely in view of recent observations of the turbulent magnetosheath that suggest reconnection of many adjacent quasi-two-dimensional magnetic islands. ${ }^{8,9}$

In the antecedent of the present work, ${ }^{29}$ it was shown that in fully developed turbulence, complex processes of reconnection locally occur. Here we further describe the statistical features of this complex scenario of reconnection events, where initial and boundary conditions are naturally imposed by the turbulence itself. In turbulence, reconnection of different-size magnetic vortices occurs simultaneously and locally. We develop systematic techniques in order to analyze reconnection in turbulence, combining classical analysis of reconnection together with statistical description of turbulence. These methods are employed to study the statistical properties of thousands of incompressible MHD reconnection events occurring at X-type neutral points at large magnetic and fluid Reynolds numbers, using direct numerical simulations with resolutions up to $16384^{2}$ grid points. We find a broad range of reconnection rates, reaching as high as $\simeq 0.3$ normalized to the root-mean-square magnetic field. A characteristic power-law distribution of rates is found relative to the geometrical aspect ratio of the reconnecting sites. We will examine how these surprising features can be brought into agreement with a generalized form of classical Sweet-Parker reconnection.

The outline of the paper is as follows. In Sec. II, the incompressible 2D MHD equations are introduced, together with the numerical method employed to solve the equations, and a global overview on fully developed turbulence at high Reynolds number. In Sec. III, a general introduction to the Hessian theory used to identify the reconnection events is presented. The statistics of the turbulent electric fields is also shown. A local description of reconnection in turbulence is presented in Sec. IV. In Sec. V, the link between reconnection and the statistical properties of turbulence is analyzed. A theoretical interpretation of the reconnection events is given in Sec. VI. Finally, in Sec VII, conclusions are given, and possible implications for turbulent plasmas in nature are discussed. Supplementary numerical tools are given in the appendix. 
TABLE I. Table of runs. In the fourth column the energy shell excited initially is reported. The last column shows the time $t^{*}$ at which the analysis has been performed (near the peak of the mean square current density $\left\langle j^{2}\right\rangle$ ).

\begin{tabular}{lcccc}
\hline \hline & Mesh points & $R_{\mu}\left(=R_{\nu}\right)$ & Initial spectrum & $t^{*}$ \\
\hline Run 1 & $4096^{2}$ & 1700 & $4 \leq k \leq 10$ & 0.4 \\
Run 2 & $4096^{2}$ & 2500 & $5 \leq k \leq 30$ & 0.2 \\
Run 3 & $4096^{2}$ & 2500 & $3 \leq k \leq 10$ & 0.7 \\
Run 4 & $8192^{2}$ & 5000 & $5 \leq k \leq 30$ & 0.3 \\
Run 5 & $8192^{2}$ & 5000 & $8 \leq k \leq 20$ & 0.3 \\
Run 6 & $16384^{2}$ & 10000 & $5 \leq k \leq 30$ & 0.3 \\
\hline \hline
\end{tabular}

\section{INCOMPRESSIBLE 2D MHD}

The 2D incompressible MHD equations can be written in terms of the magnetic potential $a(x, y)$ and the stream function $\phi(x, y)$ (uniform mass density $\rho=1$ ) as follows: ${ }^{44}$

$$
\begin{aligned}
& \frac{\partial \omega}{\partial t}=-(\boldsymbol{v} \cdot \boldsymbol{\nabla}) \omega+(\boldsymbol{b} \cdot \boldsymbol{\nabla}) j+R_{\nu}^{-1} \boldsymbol{\nabla}^{2} \omega, \\
& \frac{\partial a}{\partial t}=-(\boldsymbol{v} \cdot \boldsymbol{\nabla}) a+R_{\mu}^{-1} \nabla^{2} a,
\end{aligned}
$$

where the magnetic field is $\boldsymbol{b}=\boldsymbol{\nabla} a \times \hat{\boldsymbol{z}}$, the velocity $\boldsymbol{v}=\boldsymbol{\nabla} \phi$ $\times \hat{z}$, the current density $j=-\nabla^{2} a$, and the vorticity $\omega$ $=-\nabla^{2} \phi$. Equations (1) and (2) are written in familiar Alfvén units $^{21}$ with lengths scaled to $L_{0}$, a typical large scale length (box size is set to $2 \pi L_{0}$ ). Velocities and magnetic fields are scaled to the root-mean-square Alfvén speed $C_{A}$ and time is scaled to $L_{0} / C_{A} . R_{\mu}$ and $R_{\nu}$ are, respectively, magnetic and kinetic Reynolds numbers at scale $L_{0}$, reciprocals of kinematic viscosity and resistivity.

Equations (1) and (2) are solved in double periodic $(x, y)$ Cartesian geometry with a dealiased $(2 / 3$ rule) pseudospectral code ${ }^{45}$ We employ a standard Laplacian dissipation with constant dissipation coefficients. The latter is given small values to achieve both high Reynolds numbers and to ensure adequate spatial resolution, but is not intended to model any specific plasma kinetic process. We report results from runs with up to $16384^{2}$ grid points and $R_{\nu}=R_{\mu}=10000$. Time integration is second order Runge-Kutta and double precision is employed. A description of all the runs performed is reported in Table I.

Considering the representation of the fields in Fourier space, for a particular run (in this case run 4 , cf., Table I), the energy is initially concentrated in the shell $5 \leq k \leq 30$ (wavenumber $k$ in units of $\left.1 / L_{0}\right)$, with mean value $E=(1 / 2)\left\langle|\boldsymbol{v}|^{2}\right.$ $\left.+|\boldsymbol{b}|^{2}\right\rangle \simeq 1,\langle\cdots\rangle$ denoting a spatial average. Random phases are employed for the initial Fourier coefficients and uncorrelated, equipartitioned velocity and magnetic field fluctuations are considered. This procedure gives rise to a number of energy containing magnetic islands, of the order of 1000 for the chosen magnetic spectrum (run 4).

For the statistical analysis (see Sec. III) we consider the state of the system at $t \sim 0.3$, when the mean square current density $\left\langle j^{2}\right\rangle$ is very near to its peak value (see Fig. 1). At this instant of time the peak of small scale turbulent activity is

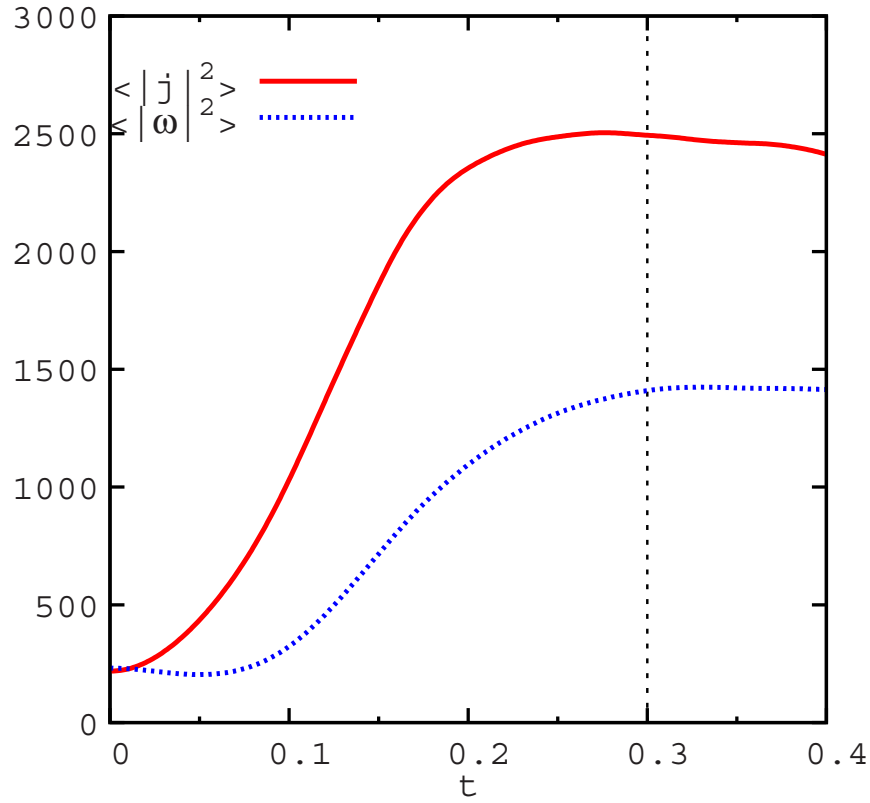

FIG. 1. (Color online) The total current density $\left\langle j^{2}\right\rangle$ (solid-red) and vorticity (blue-dashed) $\left\langle\omega^{2}\right\rangle$ as a function of time. The peak is reached around $t^{*}$ $=0.3$ (vertical black dashed line) .

achieved. The omnidirectional power spectra of the magnetic field $E^{b}(k)$ at different times are shown in Fig. 2.

When the turbulence is fully developed, coherent structures appear. They can be identified as magnetic islands that have different size and energy. At the regions between islands the perpendicular (out-of-plane) component of the current density $j$ becomes very high, as it can be seen in the color contour Fig. 3. This is related to the intermittent nature of the magnetic field ${ }^{24}$ and can be interpreted as a consequence of fast, local relaxation processes. ${ }^{46,47}$

As reported in Fig. 4, the out of plane component of the magnetic potential $a$ shows a collection of magnetic islands

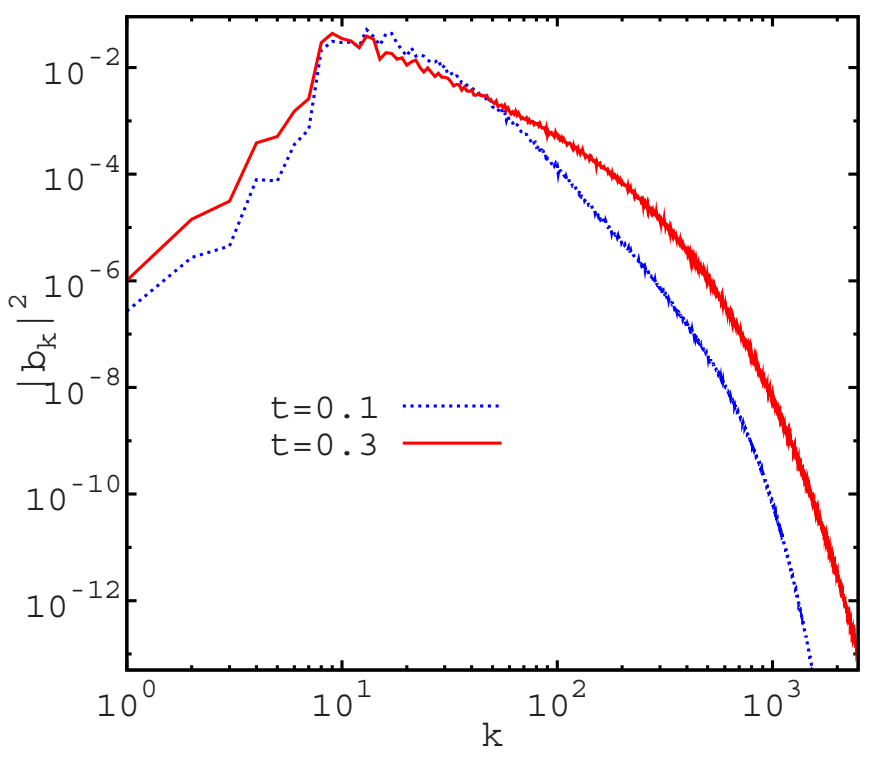

FIG. 2. (Color online) Power spectra of magnetic field at two times of the simulations. At $t^{*} \simeq 0.3$ the peak of the nonlinear activity is reached, as shown in Fig. 1. 


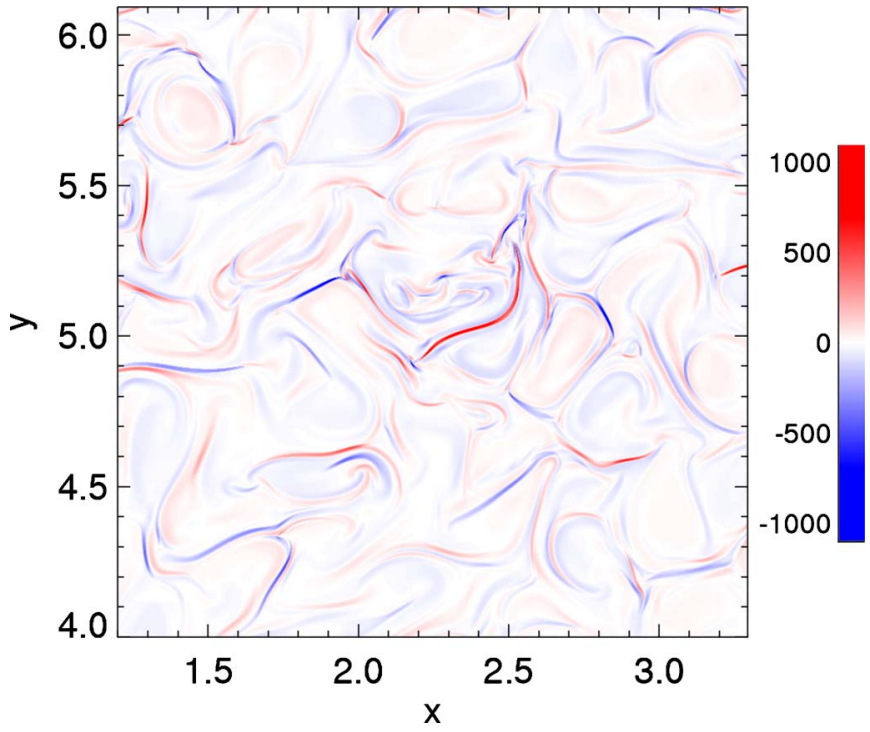

FIG. 3. (Color online) The blue-red scale color contour represents the current density $j$ in a region of the simulation box. In between magnetic islands strong peaks in the current density are present. Only about $\sim 1 / 9$ of the simulation box is shown.

having a wide distribution of sizes. In three dimensions these would correspond to flux tubes. For the large islands, the sign of $a$ in a closed field line region gives the sense of rotation of the magnetic vortex. These coherent structures interact nonlinearly, merge, stretch, connect, attract, and repulse each other. In fact the dynamics of the magnetic field in 2D MHD turbulence can be thought of as consisting largely of the interactions among these islands. Reconnection is a major element of this interaction.

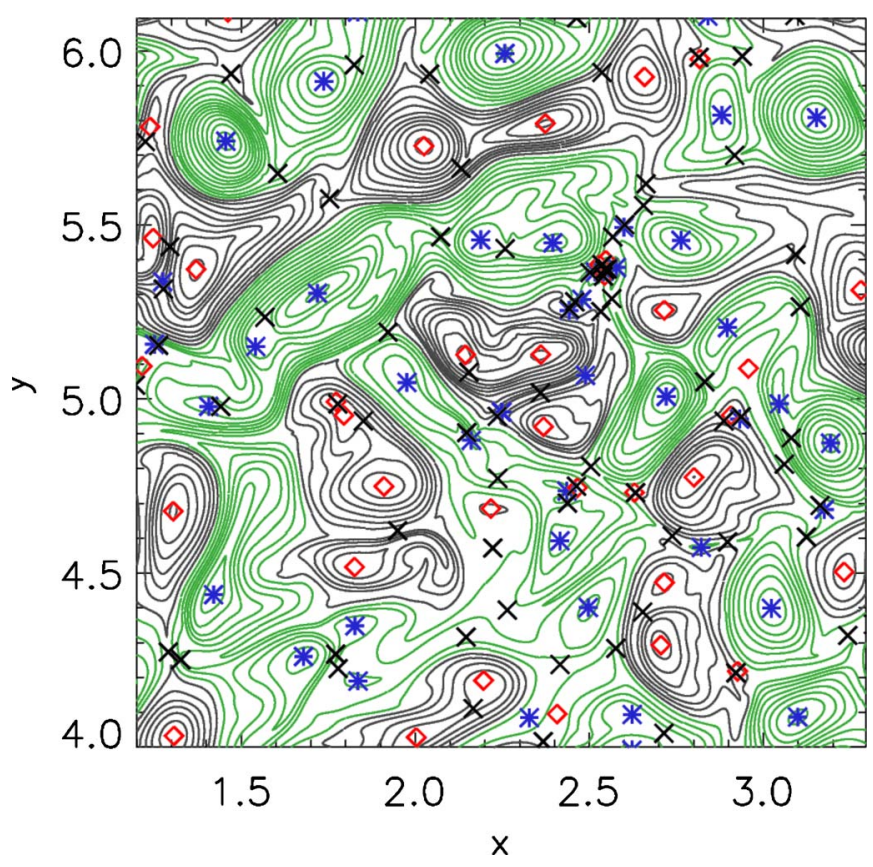

FIG. 4. (Color online) Contour plot of the magnetic potential $a$ with the position of all the critical points: O-points (blue stars for the maxima and red open diamonds for the minima) and X-points (black $\times$ ).

\section{STATISTICS OF THE RECONNECTION RATES}

\section{A. Topology of the magnetic field}

To understand reconnection in 2D turbulence, we need to examine the topography of $a(x, y)$ in detail. In particular we need to identify the neutral points. To this end we examine the Hessian matrix with the second-order partial derivatives of the potential $a,{ }^{48}$ defined as

$$
H_{i, j}^{a}(\boldsymbol{x})=\frac{\partial^{2} a}{\partial x_{i} \partial x_{j}} .
$$

At each neutral point, $\nabla a=0$, we compute the eigenvalues of $H_{i, j}^{a}$. The magnetic potential is a smooth and differentiable function and it contains key information about the magnetic field topology. If the gradient of $a$ (or equivalently, $\boldsymbol{b}$ ) is zero at some point $\boldsymbol{x}$, then $a$ has a critical point (or stationary point) at $\boldsymbol{x}$. The determinant of the Hessian at $\boldsymbol{x}$ is then called the discriminant. If this determinant is zero then $\boldsymbol{x}$ is called a degenerate critical point of $a$, which is also called a nonMorse critical point of $a$. Otherwise if it is nondegenerate, this is called a Morse critical point of $a$. Non-Morse critical points are very rare in nature. ${ }^{49}$

The following procedure can be applied to classify behavior at a nondegenerate critical point $\boldsymbol{x}$. If the Hessian is positive definite at $x$, then $a$ attains a local minimum at $x$. If the Hessian is negative definite at $\boldsymbol{x}$, then $a$ attains a local maximum at $\boldsymbol{x}$. If the Hessian has both positive and negative eigenvalues then $\boldsymbol{x}$ is a saddle point (or X-point).

Because of the complex topology of turbulence, critical points can be very close to each other. Moreover, based on a spectral representation, they are usually not located on the vertices of a chosen computational grid, so we use a second order interpolation algorithm. The problem is that a nonzero amount of energy at smallest scales is present (this can be seen from Fig. 2). This affects the precision of the interpolation technique, producing false critical points. To avoid this inconvenience we make use of a Fourier zero-padding and interpolation technique. This consists of computing the Fourier transform of $a(\boldsymbol{x})$, and, once we obtain the Fourier coefficients $\hat{a}(\boldsymbol{k})$, we copy this to an array four times bigger. The new expanded array has identical amplitudes at wave vectors present in the lower resolution representation. However it is extended by adding zeros for $k_{j} \geq\left(N_{j} / 2\right)$, where $N / 2$ the maximum $k$-vector of the original array (Nyquist frequency). Following this zero padding we inverse Fourier transform to obtain $a(x, y)$ on a higher resolution spatial grid. In this way we can generate a function that has $4 N_{x} \times 4 N_{y}$ points from the original $N_{x} \times N_{y}$. It coincides with the original function on the original grid points. Between these, on the new finer grid, it represents a trigonometric interpolation of the function. This process can require substantial computer memory, but gives results with the following desirable properties: (1) a function on a higher resolution grid with an exact Fourier expansion can be extrapolated, (2) cases in which critical points are in the same Cartesian cell are avoided, and (3) the interpolation becomes much more accurate, even if the order of the interpolation is the same (this is because the field is much smoother at the new grid size). 
After we applied the above procedure we found for run 4, for example, 631 maximum (M-points), 638 minimum (Ppoints), and 1277 saddle points (X-points). We found that the number of critical points obeys the following relation:

$$
\#\{\mathrm{M} \text { points }\}+\#\{\mathrm{P} \text { points }\}-\#\{\mathrm{X} \text { points }\}=0,
$$

which is the total number of O-points (sum of maxima and minima) equal to the number of saddle points. This rule appears to be specific to periodic geometry. In Fig. 4 we show the magnetic potential with the critical point locations, obtained with the Fourier amplification technique. In this complex picture the X-points link islands with different size and energy. In the next sections we will further explore the interesting properties of reconnection in turbulence, making use of the fields at the X-points that we have identified.

We note also that the local geometry of the diffusion region near each X-point is related to the Hessian eigenvalues,

$$
\lambda_{\max }=\frac{\partial^{2} a}{\partial s^{2}}, \quad \lambda_{\min }=\frac{\partial^{2} a}{\partial l^{2}},
$$

the larger and smaller (in magnitude), respectively, and the associated unit eigenvectors $\hat{e}_{s}$ and $\hat{e}_{l}$. The coordinate $s$ is associated with the minimum thickness $\delta$ of the current sheet, while $l$ with the elongation $\ell$. From a scaling analysis of Eq. (5), the aspect ratio of the diffusion region is well approximated by

$$
\frac{\ell}{\delta} \simeq \sqrt{\lambda_{R}}, \quad \text { where } \quad \lambda_{R}=\left|\frac{\lambda_{\max }}{\lambda_{\min }}\right| .
$$

\section{B. Properties of the electric field}

Once we obtained the position of all the critical points, a precise way to measure the reconnection rate of two islands is to compute the electric field at the $X$-point. This is related to the fact that the magnetic flux in a closed $2 \mathrm{D}$ island is computed as the integrated magnetic field normal to any contour connecting the central O-point with any other specified point. Choosing that point to be an X-point bounding the island, we find that the flux in the island is just $a$ (O-point) $-a$ (X-point) ${ }^{37}$ Flux is always lost at the $\mathrm{O}$-point in a dissipative system, so the time rate of change in the flux due to activity at the X-point is $-\partial a(\mathrm{X}$-point $) / \partial t=E_{z}$ (X-point). The latter step follows from Ohm's law expression for the electric field,

$$
\boldsymbol{E}=-\boldsymbol{v} \times \boldsymbol{b}+R_{\mu}^{-1} \mathbf{j},
$$

which in 2D involves only the out of plane component $E_{z}$ $=-(\boldsymbol{v} \times \boldsymbol{b})_{z}+R_{\mu}^{-1} j$, and which is $E_{z}(\mathrm{X}$-point $)=R_{\mu}^{-1} j$ at an $\mathrm{X}$-type neutral point.

In Fig. 5, the terms making up Ohm's law are plotted. Ohm's law electric field is dominated globally by $(\boldsymbol{v} \times \boldsymbol{b})_{z}$ (note the values on the color scales.) This fact is further reinforced by the distributions of the electric field plotted in Fig. 6, which shows the probability distribution function (PDF) of the total $E_{z}$, the advective and resistive contributions, and the values computed at the X-points. We can immediately see that the reconnection rates (electric field at the
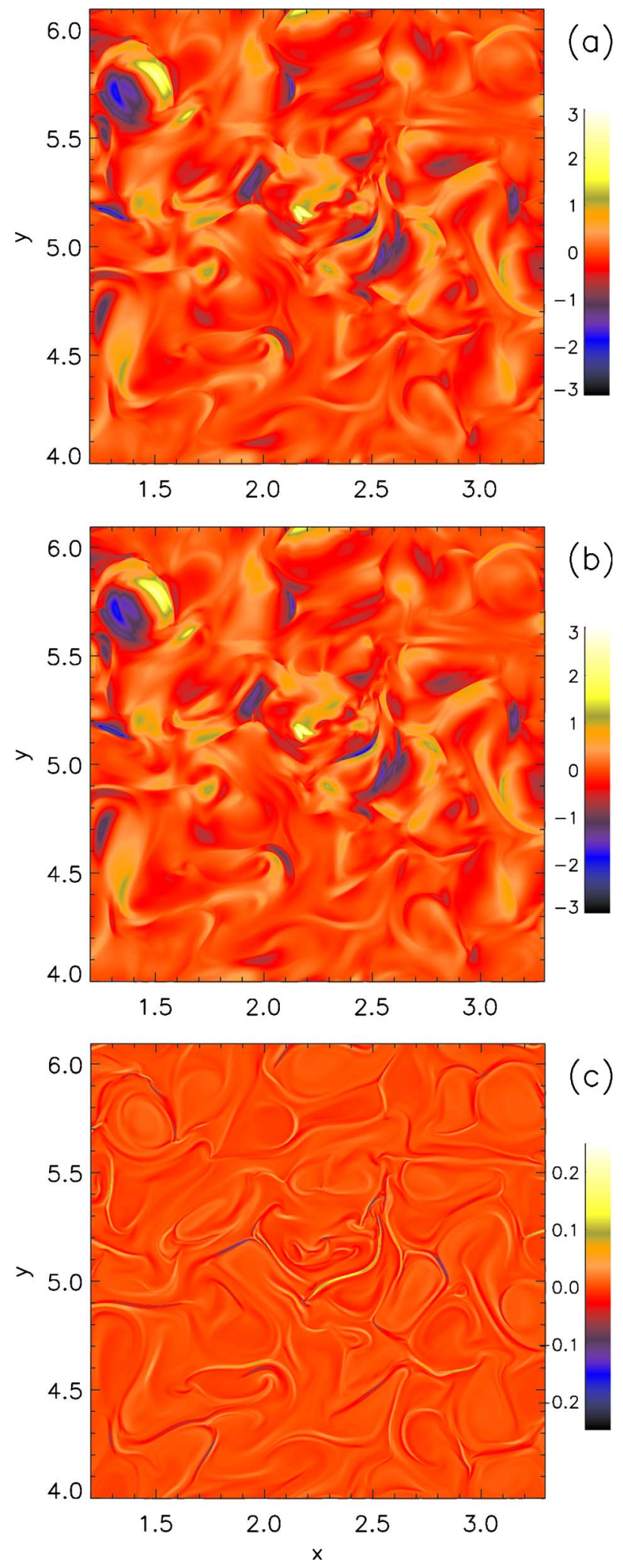

FIG. 5. (Color online) Contour plot of the out-of plane electric field contributions: (a) total electric field $E_{z}$, (b) advective term $-(\boldsymbol{v} \times \boldsymbol{b})_{z}$, and (c) diffusive term $R_{\mu}^{-1} j$ term.

$\mathrm{X}$-points) are very narrow compared to the total electric field. We will further discuss this important feature of turbulent reconnection in the final section of the paper.

Also clear from Figs. 5 and 6 is that the total electric 


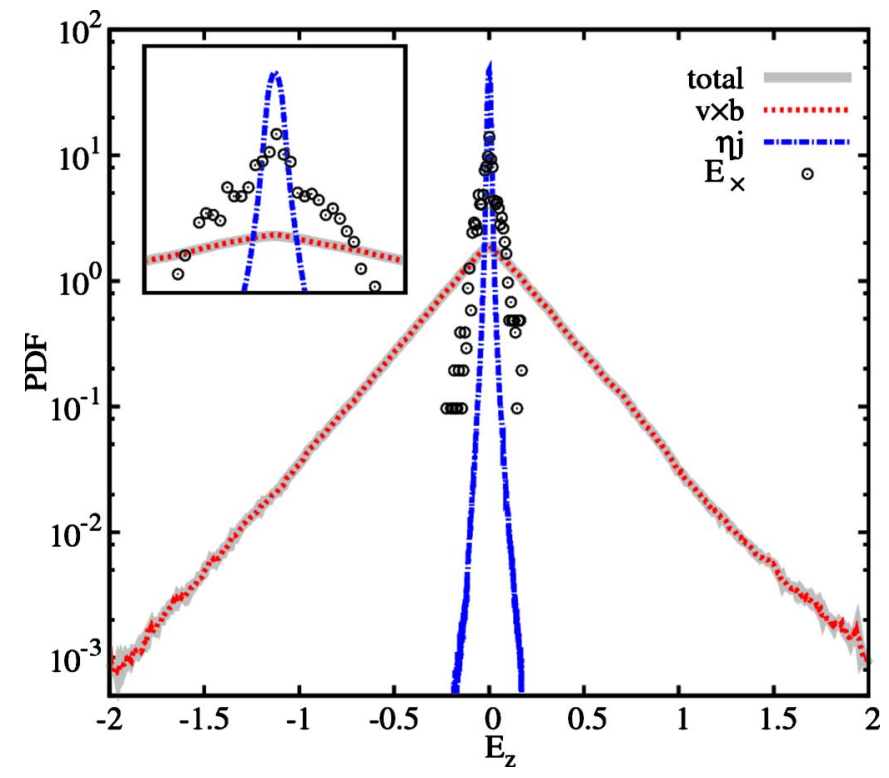

FIG. 6. (Color online) PDFs of (gray) total $E_{z}$ given by Ohms' law, Eq. (7); (red dotted) advective component $-(\boldsymbol{v} \times \boldsymbol{b})_{z}$; (blue dot-dashed) diffusive component $R_{\mu}^{-1} j$; (black open circles) reconnection rates $E_{z}$ (X-point). Inset: zoom of the core of these distributions.

field is essentially due to the term $\boldsymbol{v} \times \boldsymbol{b}$, which is the electric field produced by fluid plasma motions. The diffusive electric field $R_{\mu}^{-1} j$ (note that we use $\eta$ or $R_{\mu}^{-1}$ interchangeably), is very small and with a much narrower distribution. ${ }^{25}$ The resistive $R_{\mu}^{-1} j$ contribution is non-Gaussian and gives information about the intermittent nature of MHD turbulence. It is very interesting that the tails of the distribution of the reconnection rate are narrower than the tails of the advective electric field, but are wider than the tails of the diffusive electric field. Moreover the strongest current structures are associated with the strongest values of the reconnection electric field. This is a simple manifestation of the fact that the strongest current concentrations are typically found near the X-points. This confirms that turbulent reconnection is closer to a nonlinear instability rather than to a simply diffusive problem. For values randomly chosen from each population (not at a single point), we can see that typically the largest magnitudes are those of the inductive field, those least in magnitude will be the resistive field, and the reconnection electric field values are intermediate. For any even moment statistic $M(x)$ $=\left\langle x^{2 n}\right\rangle$ (integer $n$ ), we have

$$
M(\boldsymbol{v} \times \boldsymbol{b})>M\left(E_{\times}\right)>M\left(R_{\mu}^{-1} j\right) .
$$

The shape of the distribution of the advective component $-(\boldsymbol{v} \times \boldsymbol{b})_{z}$, which resembles an exponential distribution, is typical of both solar wind plasmas and numerically simulated strong MHD turbulence. ${ }^{50,51}$

Following the discussion above, the reconnection rates are computed as the rate of change of the magnetic flux
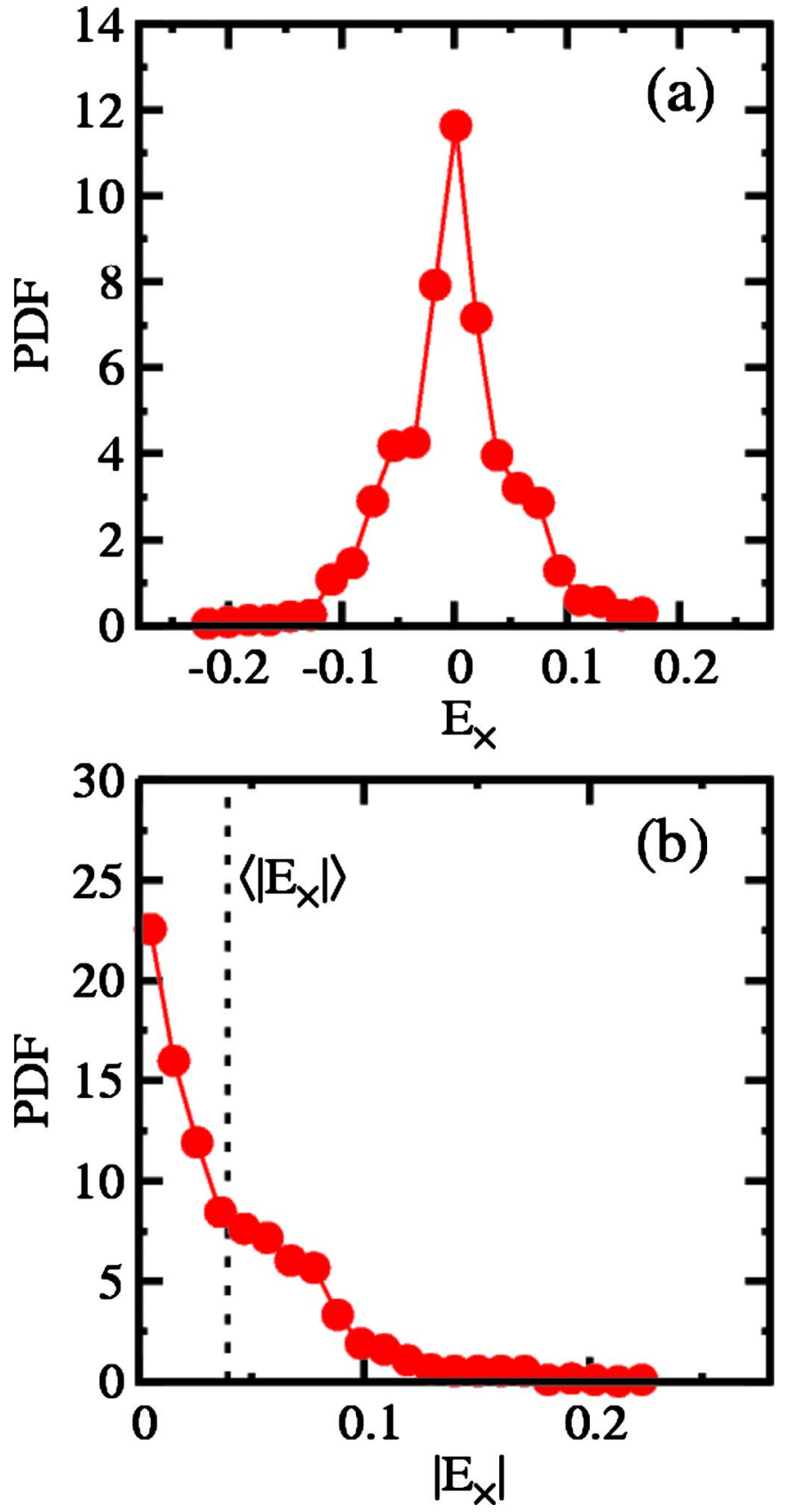

FIG. 7. (Color online) (a) PDF of the normalized values of $E_{z}$ (X-point) (reconnection rate), (b) PDF of $E_{z}(\mathrm{X}$-point). Vertical dotted line in (b) represents the mean value of the distribution.

through $\partial a / \partial t$, and their distribution is further examined in Fig. 7. These rates are computed using Eq. (7) at the saddle points, where

$$
\frac{\partial a}{\partial t}=\left(R_{\mu}^{-1} j\right)_{\times}=-E_{\times}
$$

and $E_{\times}$is an abbreviation for the electric field measured at the (X-point) saddle point, and similar notation indicates that the Ohmic electric field is evaluated at the same X-point.

The PDF of the electric field at the X-points is quite broad and peaked around the zero value. Here the reconnection rates have been normalized to the mean square fluctuation $\delta b_{\mathrm{rms}}^{2}$, appropriate for Alfvènic turbulence. In Fig. 7(b) 


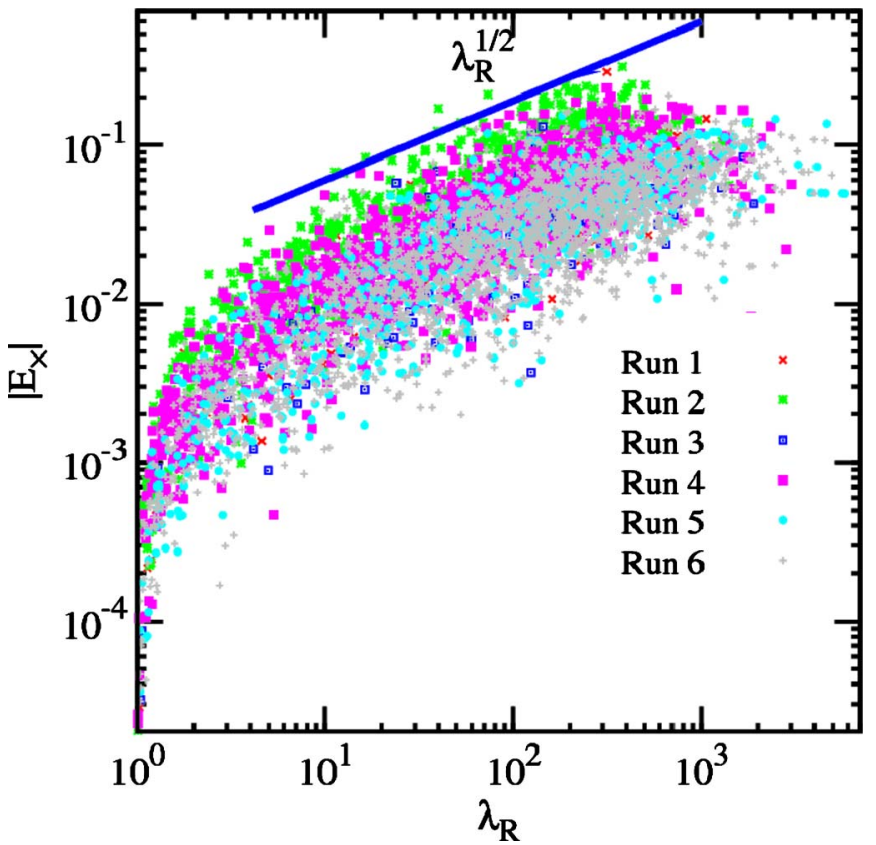

FIG. 8. (Color online) The relation between the reconnection rate (the electric field at the X-point) and the geometry of the reconnection region (the ratio of the eigenvalues). The presence of a power-law fit (blue dotted line) demonstrates that there is a relation between the reconnection rate and the geometry of the diffusion region. Note that $E_{\times} \sim \ell / \delta$.

the PDF of the absolute value of the reconnection rate is shown. The mean value of the reconnection rate is $\simeq 0.04$, with strong variations from the average, that is values are found in the range $\left|E_{\times}\right| \in\left[10^{-6}, 0.3\right]$.

In terms of the global parameters this observed range of reconnection rates varies from very slow to fast. For $R_{\mu}$ $=10000$ the global Sweet-Parker rate would be computed as $\sim 0.01$. In this sense the typical reconnection rate in turbulence is found to be far higher than what is expected based on a simple global application of the Sweet-Parker rate $E_{\times}$ $\sim R_{\mu}^{-1 / 2}$. We now examine more details of how these rates arise.

In the case in which the reconnection is in a stationary state, the rate depends on the aspect ratio defined by Eq. (6). In Fig. 8 it is shown that there is a relation between the stronger reconnection rates and the geometry of the reconnection region. In fact the electric field values for the stronger reconnection regions (see figure) satisfy the following approximate scaling relation:

$$
E_{\times} \sim \frac{\ell}{\delta}=\sqrt{\lambda_{R}}
$$

where $\lambda_{R}=\left|\lambda_{\max } / \lambda_{\min }\right|$.

The last relation seems to be counter intuitive, because in the classical model of steady state reconnection $E_{\times}$ $\sim \delta / \ell$. We will return to this issue in more detail in Sec. VII.

Another important relation links the eigenvalues of the Hessian matrix with the current density at the X-points:
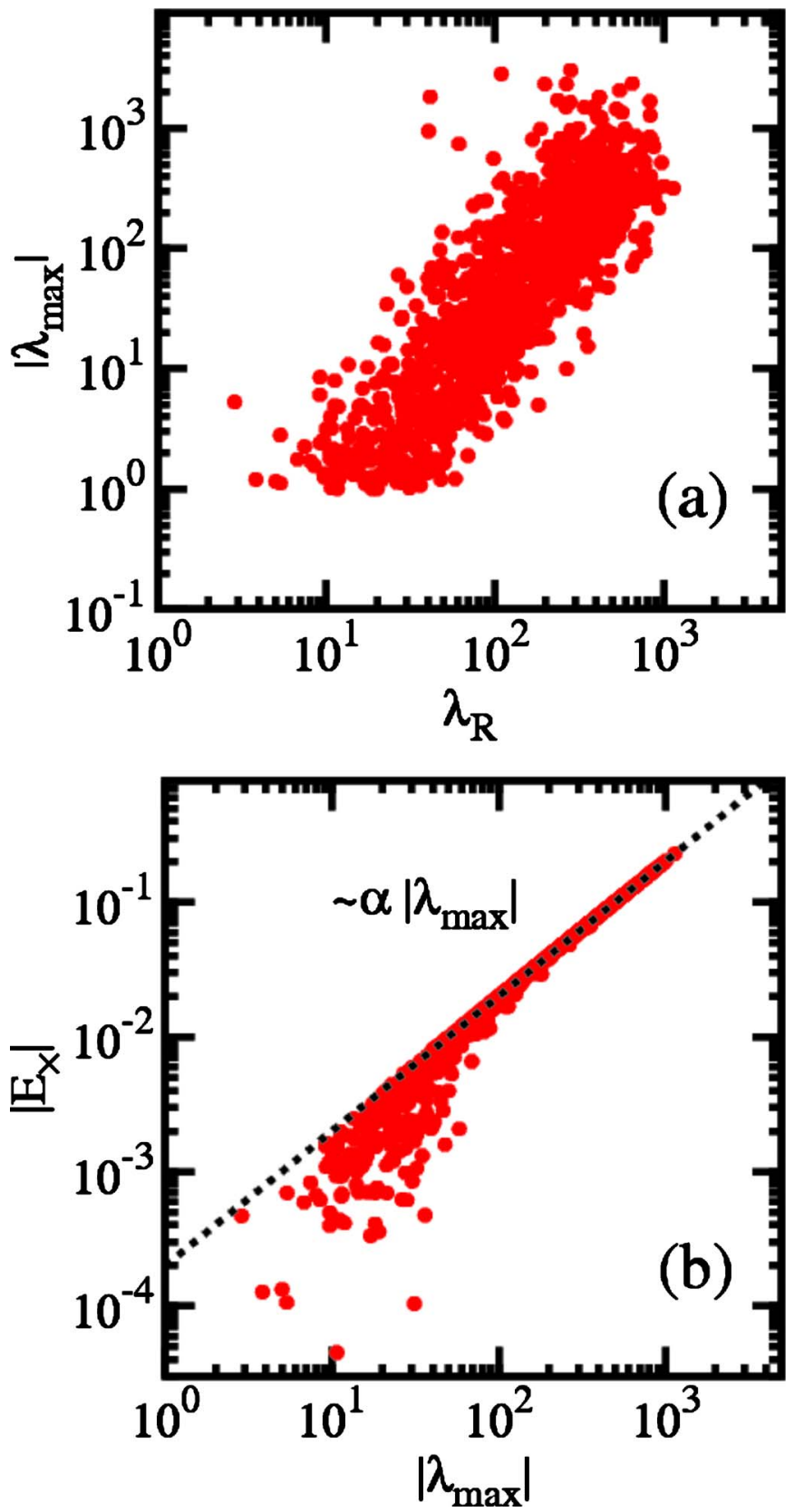

FIG. 9. (Color online) Panel (a): scatter plot of the bigger eigenvalue $\lambda_{\max }$ vs the ratio of eigenvalues $\lambda_{R}$. In panel (b) a numerical test that verify Eqs. (9) and (12) is shown. The slope $\alpha$ of the fit (black dotted) is very close to the value of the resistivity $R_{\mu}^{-1}$.

$$
j_{\times}=-\left(\lambda_{\max }+\lambda_{\min }\right),
$$

because at the high-rate reconnecting regions $\lambda_{\max } \gg \lambda_{\min }$. For those cases we find

$$
j_{\times} \simeq-\lambda_{\max } .
$$

Figure 9(a) shows a scatter plot of the larger of the two eigenvalues $\lambda_{\max }$ versus $\lambda_{R}$. This result is another indication (less direct) that the sites with highest reconnection rate are the ones with $\delta \ll \ell$. In Fig. 9(b), a numerical test that verifies the approximation in Eq. (12) is shown. The slope of the fit in this figure is very close to the value of the resistivity $R_{\mu}^{-1}$, cf. Eq. (9). 


\section{Rapid reconnection and phase randomization}

The power-law fit in Fig. 8 suggests that Eq. (10) holds only for the fastest reconnection events. For slowly reconnecting regions there is no clear scaling. We now show that the collection of slowly reconnecting (or even nonreconnecting) X-point regions is associated with a distribution of magnetic fields that is Gaussian, whereas the strongly nonlinear interacting ingredient of the fluctuations would be expected to produce intermittent structure and non-Gaussian statistics. In order to select the most relevant events we need to establish a threshold for the X-point electric field, and for the aspect ratio of the eigenvalues. From Fig. 8 it seems reasonable that for $\left|E_{\times}\right| \gg 10^{-2}$ (or $\lambda_{\max } / \lambda_{\min } \gg 100$ ) a scaling region is present. We now employ a phase-randomizing procedure. This kind of technique, used in turbulence studies, ${ }^{46,52}$ consists of taking the Fourier coefficients of a variable, in this case $a(\boldsymbol{x})$, and forming from these a new function $a^{\text {Gauss }}(\boldsymbol{x})$ that has the same power spectrum, but with random phases. The new $a^{\text {Gauss }}$ has no coherency, a property that comes from the nonlinear nature of MHD and is hidden in the phases of the Fourier coefficients expansion. The result of the phase randomization can be seen in a section of the simulation domain shown in Fig. 10, where the level contour of $a$ and $a^{\text {Gauss }}$, within the respective critical point positions, are compared. The number of critical points dramatically increases in the Gaussianized variable. This is due to the fact that gradients are more uniformly distributed, leading to a more complex topology of the field. Even the randomized case satisfies the critical point sum rule given by Eq. (4).

This technique identifies the features of the reconnection rate distribution that are due to the Gaussian and nonGaussian features of the fluctuation fields. According to the procedure we adopted for the unaltered turbulent simulation, when $\left|E_{\times}\right|>0.01$ and $\lambda_{\max } / \lambda_{\min }>100$, we find a direct relation between the reconnection rate and the geometry of the diffusion region is present. These are the strong reconnection sites. We also computed the reconnection rate distribution for the Gaussianized field. As it can be seen from Fig. 11, the reconnection rates of the incoherent randomized magnetic field are on average much weaker than for the original case. In fact the part of the distribution where we found the strongest reconnection sites and the scaling relation with aspect ratio is completely absent in the Gaussianized case. Evidently, the nonlinear, intermittent nature of MHD is responsible for the faster reconnection rates that are observed.

Before continuing the exposition of the results, we remark on our procedures for verification, as we have found that the stable and accurate determination of the distribution of reconnection rates requires considerable care, especially with regard to spatial resolution. Numerous test cases were examined in this regard. The numerical results shown were verified in a number of ways: we compared runs with different time steps and spatial resolutions, checked that the dissipation scale is resolved, and examined field lines at the grid scale for adequate microscopic smoothness. We also checked for proper scalings of higher order statistics (see Ref. 53) and
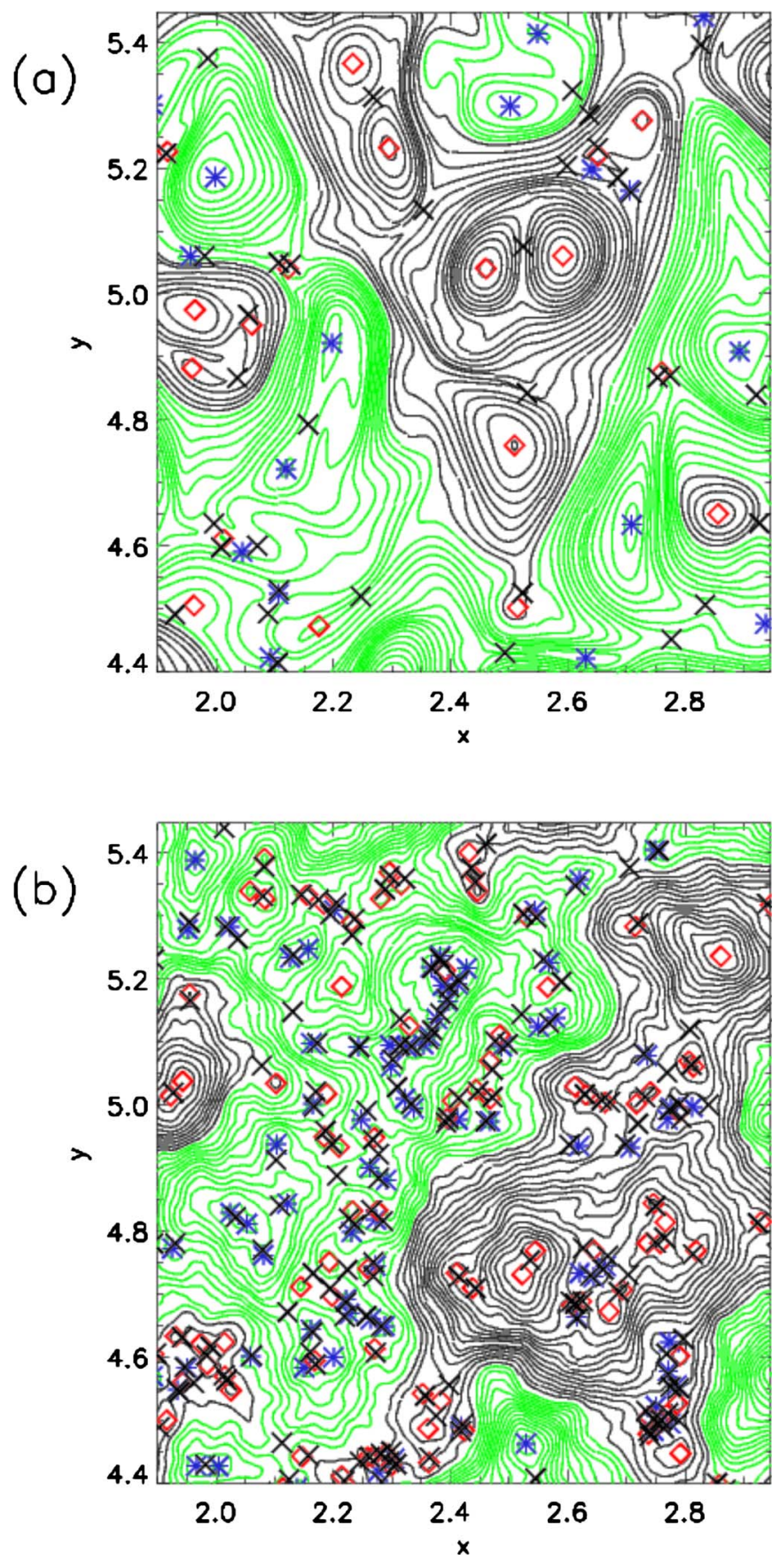

FIG. 10. (Color online) Comparison between $a$ (a) and $a^{\text {Gauss }}$ (b) in a small section of the simulation domain. On each plot the critical points are represented. The notation is the same as Fig. 4. One sees many more critical points in the Gaussianized, incoherent field, even though the magnetic energy spectra are identical.

for well behaved distributions of reconnection rates. For example, as suggested by the results above, Gaussianization of the small scales is an important type of error that can influence the reconnection rates. Lack of adequate resolution can cause small scale thermalization of the phases that are crucial for computing correct reconnection rates. In the future we will provide further detail on these underlying numerical analysis issues. 


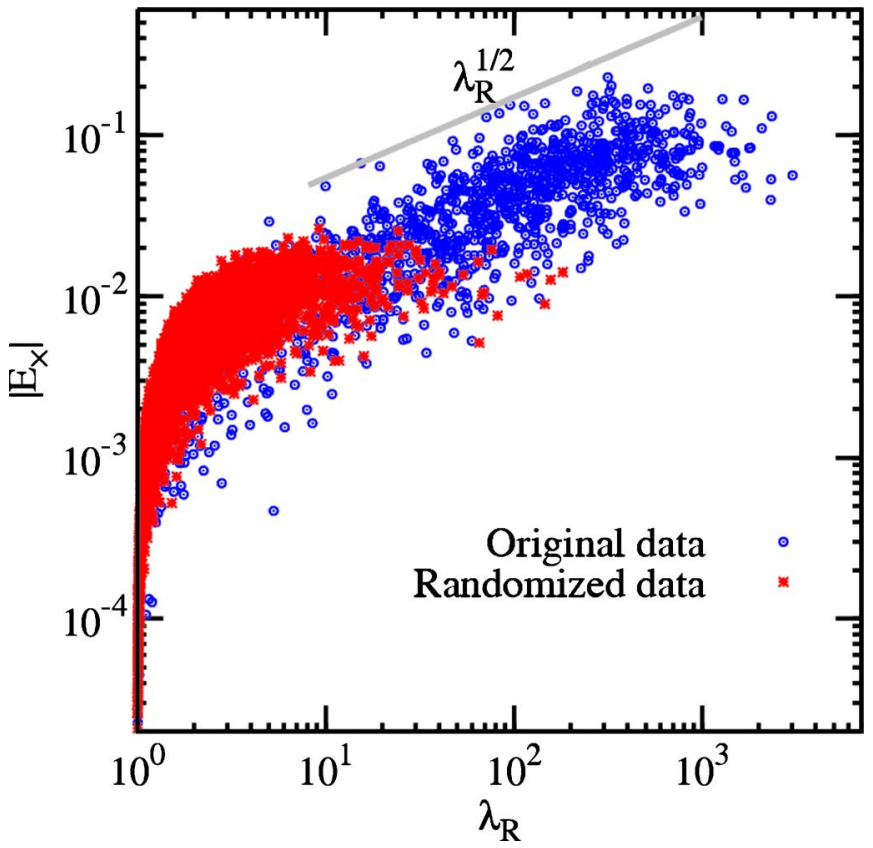

FIG. 11. (Color online) Same as Fig. 8, within the relative "randomized" reconnection rate (red starts). See the text for more details.

\section{A LOCAL DESCRIPTION OF RECONNECTION IN TURBULENCE}

\section{A. The distribution of islands}

Now we will take a closer look at the reconnection sites. We want to identify the main features of reconnection in turbulence, trying to link it with 2D MHD turbulence properties. Because of the complexity of the geometry we will focus only on the $\mathrm{X}$-lines with higher reconnection rates, identified using the comparison between the original and Gaussianized fields as described above.

In turbulence, magnetic islands of a range of sizes are present. Sometimes multiple islands simultaneously reconnect. Every island (or every cluster of reconnecting islands) is embedded in a bigger island that, at the same time, is merging with other magnetic islands. The picture of turbulence is rather complicated, but it is possible to systematically identify the largest islands, and the islands with the highest reconnection rates. To assist in this analysis we made use of a cellular automata technique (see Appendix), which helps us to find, for each selected island, the last closed magnetic surface (LCMS). In effect this associates with each O-point or magnetic axis, a unique nearby X-point, namely, the one that is "closest" in terms of magnetic flux, although not necessarily closest in distance.

In laminar theories of steady state reconnection usually the reconnecting islands are assumed to be symmetric. In order to roughly test the validity of the symmetric approximation in turbulence, we computed the shape of the LCMS for some of the fast reconnecting events. The result is shown in Fig. 12, where different closed area surfaces are plotted together with contours of $a$. Most of the reconnecting islands are not symmetric, that is the islands that are reconnecting

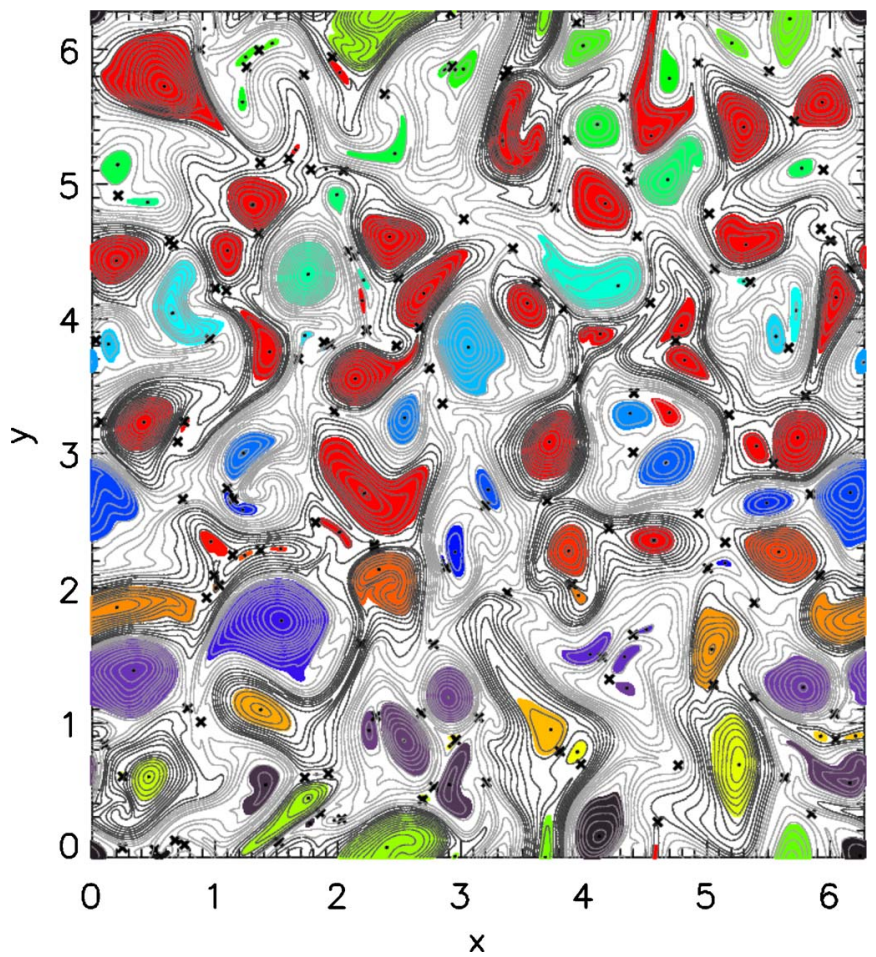

FIG. 12. (Color online) Contour lines of the magnetic potential, together with color-shaded areas identified by the cellular automata technique. Each colored closed region identifies a flux tube, defined by its last closed flux surface. The choice of color is only for convenience. The X-points are shown by the symbol "X."

have different extension, shape, and amount of energy. This suggests that the symmetric laminar theory of steady state reconnection may not be appropriate to describe the strongest reconnection sites found in turbulence.

\section{B. Characteristics of diffusion regions}

We need at this point to find a methodology to quantitatively characterize every reconnection region and extrapolate important information, including $\delta$ and $\ell$. Since we know the ratio of the eigenvalues obtained from the Hessian matrix analysis, the problem reduces to find just one of these lengths, such as the current sheet thickness $\delta$. The elongation of the current sheet $\ell$ can then be obtained using Eq. (6). We build a system of reference that has its origin at the X-point. The eigenvectors of the Hessian matrix, defined by Eq. (3), identify the directions associated with inflow $(s)$ and outflow (l) regions. These eigenvectors $\boldsymbol{e}_{s}$ and $\boldsymbol{e}_{l}$ correspond to the eigenvalues in Eq. (5), and refer to the directions of greater curvature $\left(\boldsymbol{e}_{s}\right)$, and lesser curvature $\left(\boldsymbol{e}_{l}\right)$, of the magnetic potential $a$. Once we compute these eigenvectors, it is possible to construct this system of reference for each reconnection region, given by the unit vectors $\left\{\hat{\boldsymbol{e}}_{s}, \hat{\boldsymbol{e}}_{l}\right\}$.

In Fig. 13(a) an example of the current density profile along the $s$-direction is shown. A consequence of the asymmetric nature of turbulent reconnection implies that in most of the cases the current density has a peak not centered precisely on the X-point. ${ }^{54}$ We call $b_{t}(s)$ and $b_{n}(s)$ the normal 

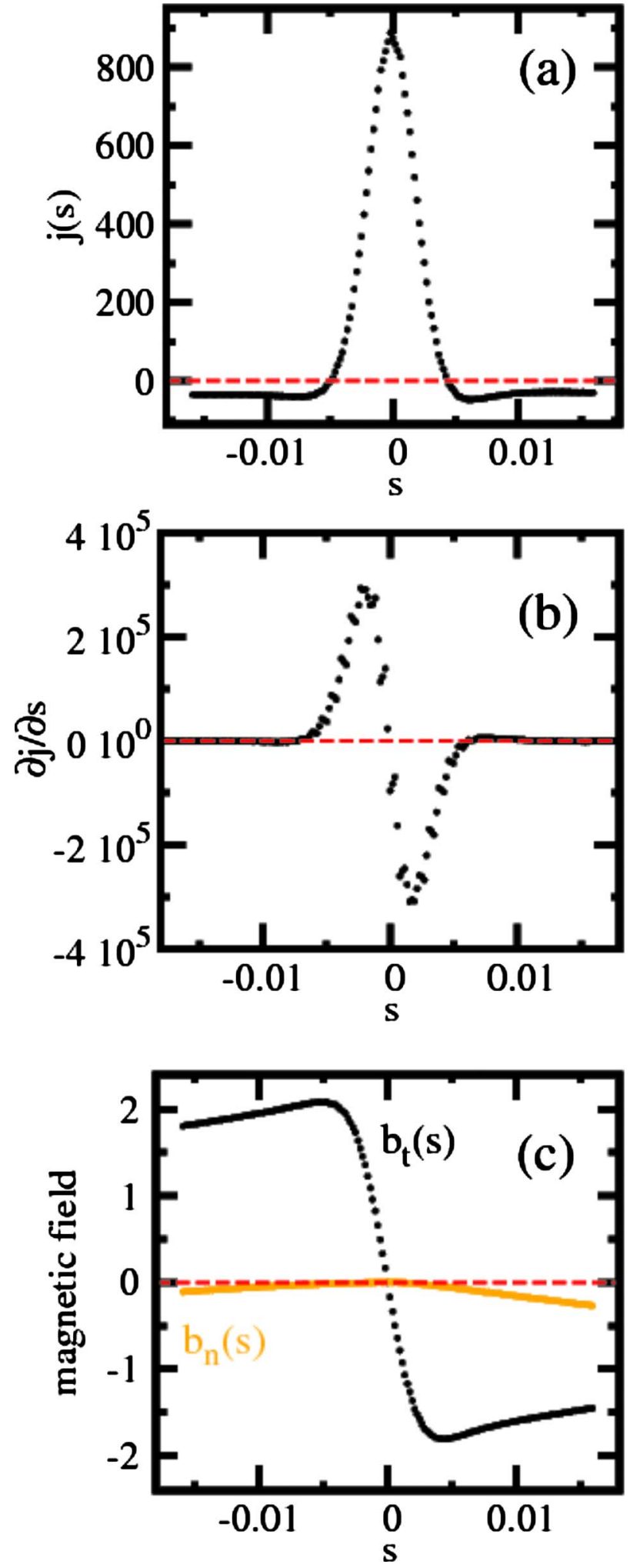

FIG. 13. (Color online) Profiles of the current density (a) and its derivative $\partial j / \partial s(\mathrm{~b})$ in the vicinity of a X-point. The X-point is located at $s=0$. In the panel (c) the tangential $\left(b_{t}\right.$, black circles) and the normal $\left(b_{n}\right.$, orange bullets) components of the magnetic field are shown. Horizontal dashed (red) lines represent zero values.

and the tangential component of the magnetic field, respectively. These components are obtained by projecting the inplane magnetic field into the system of reference given by $\left\{\hat{\boldsymbol{e}}_{l}, \hat{\boldsymbol{e}}_{s}\right\}$, that is
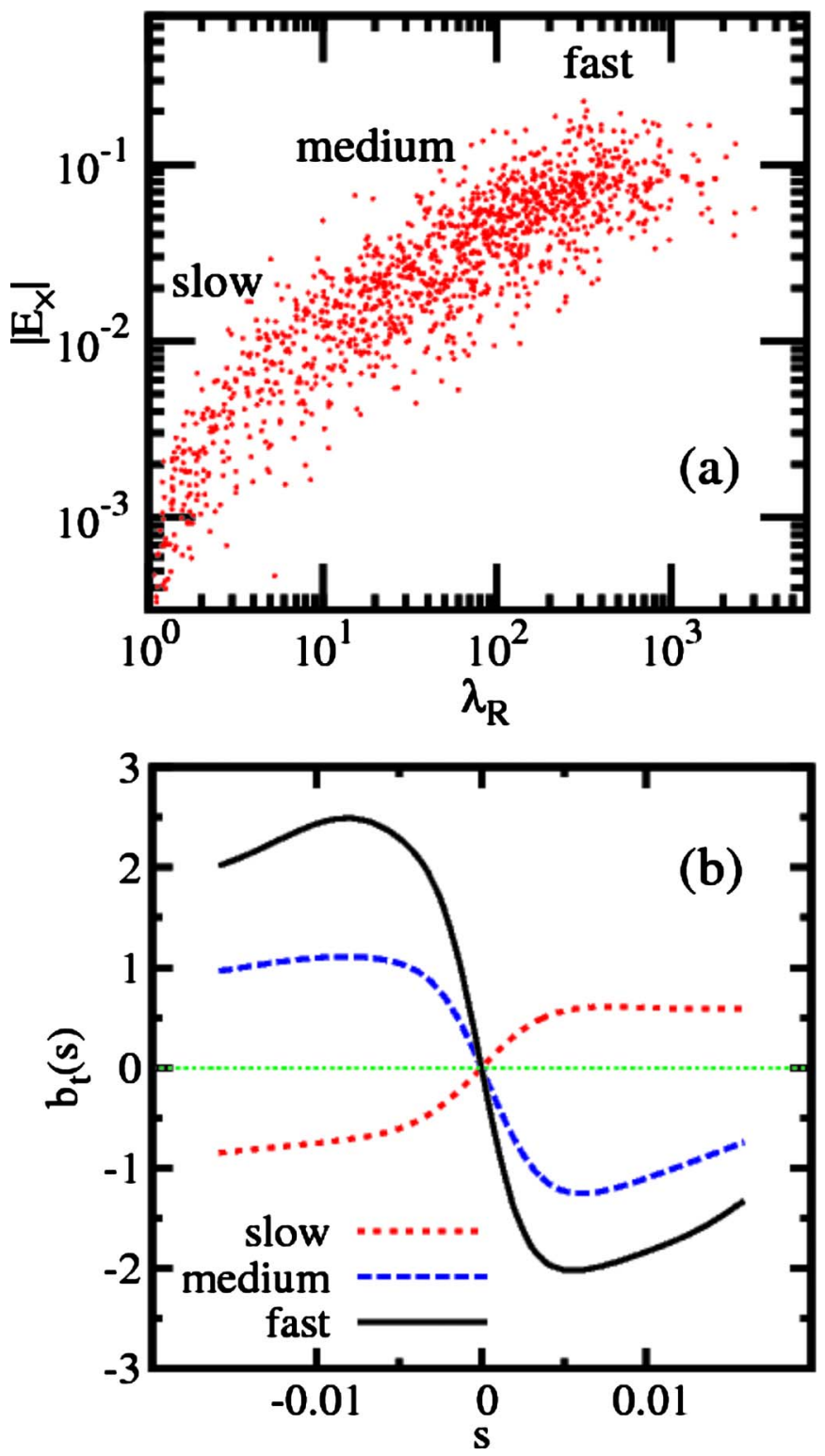

FIG. 14. (Color online) Comparison of the magnetic configuration for $\mathrm{X}$-points in different ranges of reconnection rate, identified as slow, medium, and fast cases. These ranges are shown in the upper panel (same reconnection rate distribution as Fig. 8). In the lower panel are three examples: slow (red dashed line), medium (blue dashed line), and fast (full black line) reconnection. The horizontal dashed (green) line represents the zero value.

$$
\begin{aligned}
& b_{t}=\hat{e}_{l} \cdot \boldsymbol{b}, \\
& b_{n}=\hat{e}_{s} \cdot \boldsymbol{b} .
\end{aligned}
$$

In Fig. 14 we compare some example $b_{t}$ profiles, for three selected $\mathrm{X}$-point regions, choosing in particular regions with widely different reconnection rates. The overall widths are not greatly different, but there is a significant systematic difference exemplified by these cases: the strongest reconnection events have a magnetic field that reaches a maximum and then decreases going far from the saddle point. This gives rise to much steeper gradients of the field near the neutral point. In the slowly reconnecting cases the magnetic field increase is gradual and monotonic. This behavior is 
very different from what is usually found in standard laminar reconnection studies, where, for initial value problems, generally a uniform magnetic field is imposed away from the diffusion region. There are also well studied cases in which the field attains a maximum at some offset from the X-linethis is known as flux pile-up. ${ }^{55,56}$ This also occurs in some island coalescence problems. ${ }^{57,58}$ Flux pile-up reconnection has been observed recently in an experiment on colliding flux ropes. ${ }^{59}$

At this point we need to find the width $\delta$. Because of the asymmetry of the problem, we compute the total width of each current peak as the sum of a contribution $\delta_{1}$ from the right side (region 1) and $\delta_{2}$ from the left side (region 2). Then we sum these to compute the width, employ the known eigenvalue ratio to obtain the length of each reconnection region as

$$
\begin{aligned}
& \delta=\delta_{1}+\delta_{2}, \\
& \ell=\delta \sqrt{\frac{\lambda_{\max }}{\lambda_{\min }}} .
\end{aligned}
$$

Specifically, the values of $\delta_{1,2}$ are found by assuming that the magnetic field, close to the X-point can be approximated as a hyperbolic tangent.

We interpolate the current density $j$, along the inflow coordinate $s$, using the following parametric functions:

$$
\begin{aligned}
& f_{\text {left }}(s)=A_{1} \operatorname{sech}^{2}\left(\frac{s-s_{0}}{\delta_{1}}\right)+C_{1}\left\{s<x_{0}\right\}, \\
& f_{\text {right }}(s)=A_{2} \operatorname{sech}^{2}\left(\frac{s-s_{0}}{\delta_{2}}\right)+C_{2}\left\{s \geq x_{0}\right\},
\end{aligned}
$$

being $A_{1,2}$ the amplitudes (they are proportional to the magnetic field inside the two islands), $s_{0}$ the position of the current peak, and $C_{1,2}$ local constants. For each X-point the fit has been optimized by an iteration procedure in order to minimize the error of the interpolation. ${ }^{60}$ Using the above procedure, the lengths of the diffusion region $(\delta$ and $\ell$ ) and the up-stream tangential magnetic fields $\left(b_{1}\right.$ and $\left.b_{2}\right)$ have been found [note we suppress the index " $t$ " that designates the tangential component in Eq. (13)].

A simple way to initialize the fit procedure is to roughly estimate the $\delta_{1,2}$ by using $\partial j / \partial s$ in the following way. As can be seen from Fig. 13(b), the width of the current peak approximately corresponds to the distance between the two peaks in $\partial j / \partial s$. Beginning with this estimation of $\delta_{1,2}$, we then optimize the fit of Eq. (15) by iteration. Note that we are interested in the diffusion region, and not in the details of what happens at larger values of $s$. The result of this procedure gives a very good interpolation of the current density at the X-points, as it can be seen from the example in Fig. 15.

Following an application of the above procedure to all the strong reconnection sites, we obtain statistics that characterize the shape and size of that population of sites. In Fig. 16 we show an example of a particular box placed on its reconnection site with width $\delta$ and length $\ell$, superposed on

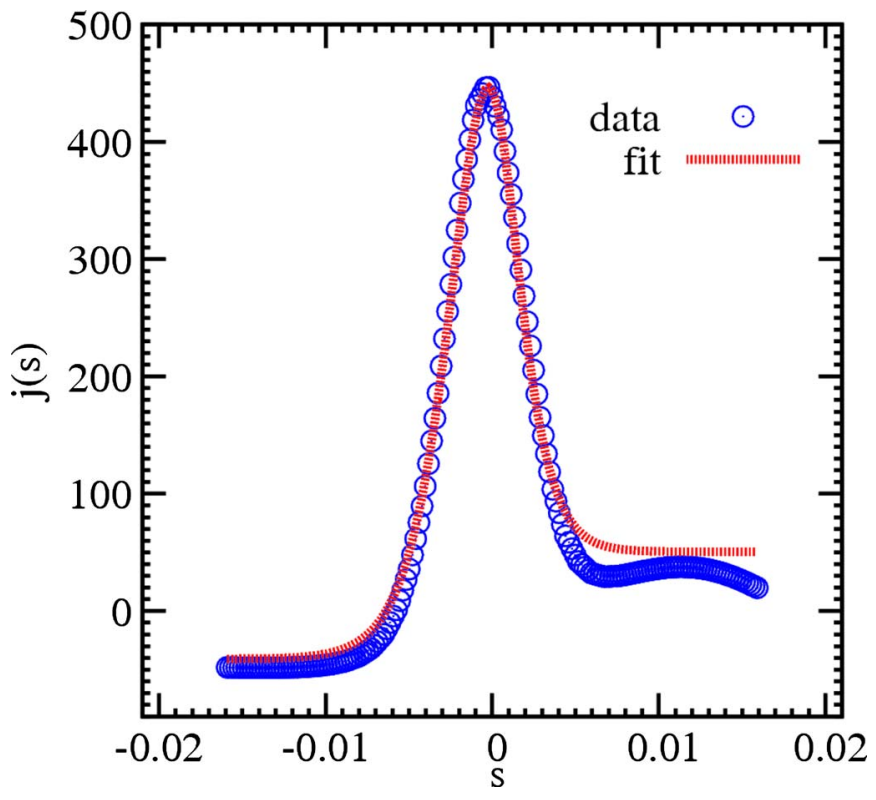

FIG. 15. (Color online) Optimized fit (red dashed line) of the current density $j(s)$ (blue circles) in the vicinity of an X-point. The best fit function is given by Eq. (15). The fit-algorithm interpolates the function in the vicinity of the $\mathrm{X}$-point, since we want to estimate the diffusion region width $\delta$ (see text).

the $a$ contours and current density near the reconnection region. The larger length $\ell$ is the one associated with the extension of the current sheet (along $\hat{\boldsymbol{e}}_{\ell}$ ).

The values of $\delta$ and $\ell$, for every reconnecting event that exceed the threshold $\left|E_{\times}\right|=0.01$, are reported in Fig. 17. We computed the mean values for these two lengths and we found $\langle\delta\rangle \simeq 5 \times 10^{-3}$ and $\langle\ell\rangle \simeq 0.1$. The values of $\ell$ show much more spread than $\delta$. The distributions of these lengths are clustered around certain values. We will now examine how these lengths are related to turbulence length scales.

\section{THE LINK BETWEEN RECONNECTION AND TURBULENCE}

The system is in a regime of fully developed turbulence. The power spectrum in Fig. 18 may be divided into a large scale wavenumber range $(1 \leq k \leq 10)$, an inertial range (20 $\leq k \leq 80)$, and a dissipation range $(100 \leq k \leq 500)$. The spectrum and correlation functions give information of relevance to the length scales associated with the turbulence. The present goal is to look for possible links between the reconnection geometry and the statistical properties of turbulence. The wave vectors corresponding to the average width $\delta$ and the length $\ell$ are given by

$$
k_{\delta}=\frac{1}{\delta}, \quad k_{\ell}=\frac{1}{\ell} .
$$

As can be seen in Fig. 18, the diffusion width is related to the dissipation scale, or the upper end of the inertial range, while $k_{\ell}$ is of the order of the wavenumber at which the spectrum peaks. The distribution of $\delta$ and $\ell$, together with their mean values are reported in Fig. 19(a).

In order to get more information about these associations we computed the autocorrelation function of the magnetic field. The correlation length is defined as 

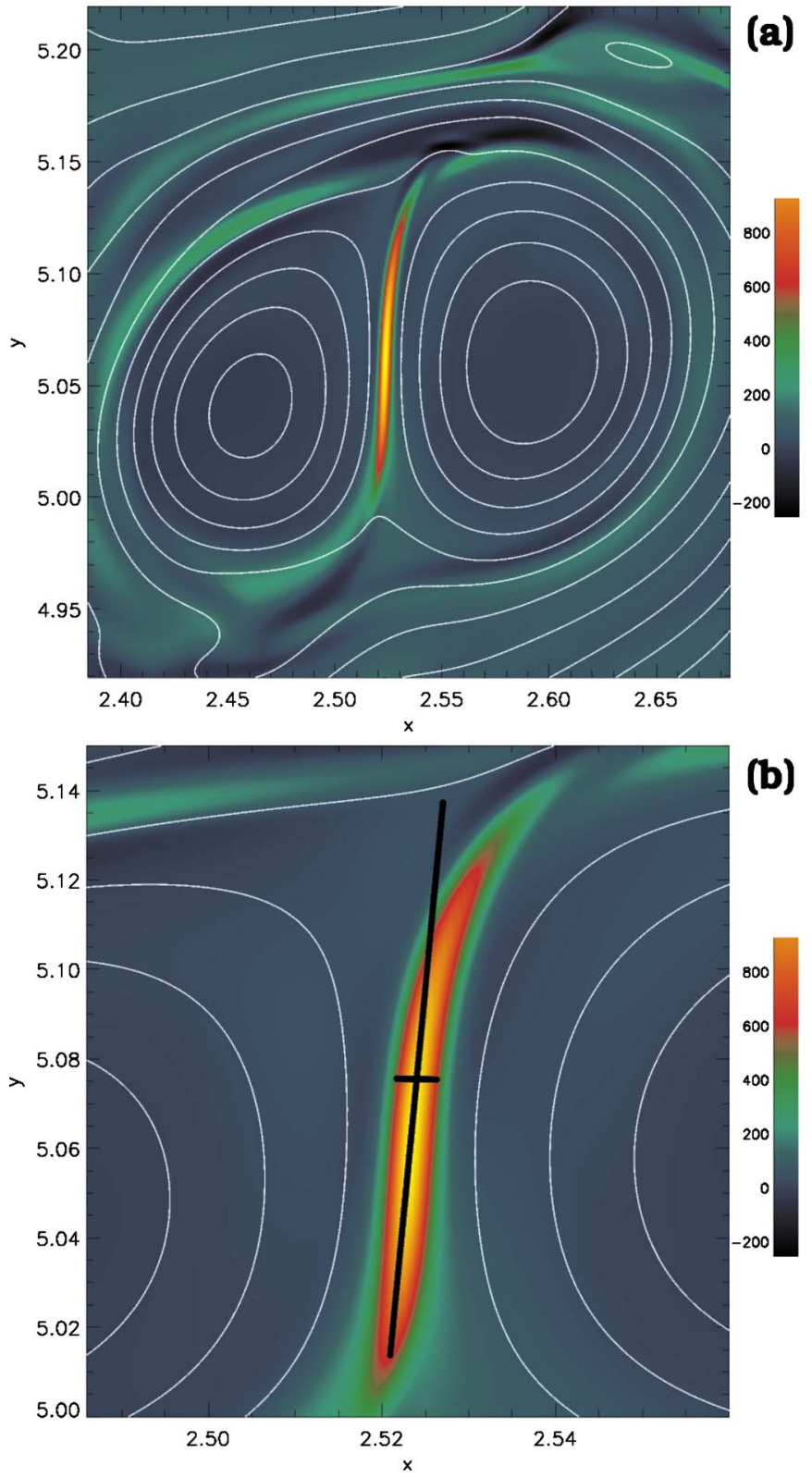

FIG. 16. (Color online) (a) Contour lines of $a$ (white solid lines) together with the current density $j$ (color scale) in a region of reconnecting islands. (b) Zoom into the diffusion region with the characteristic lengths $\ell$ and $\delta$ (black cross). Note that $\delta \ll \ell$ in this case.

$$
\lambda_{C}=\int_{0}^{*} C(r) d r
$$

where

$$
C(\mathbf{r})=\frac{\langle\boldsymbol{b}(\boldsymbol{x}+\mathbf{r}) \cdot \boldsymbol{b}(\boldsymbol{x})\rangle}{\left\langle b^{2}\right\rangle},
$$

where the direction of displacement $r$ is arbitrary for isotropic turbulence in the plane, and the upper limit is unimportant if the distant eddies are uncorrelated. The correlation length $\lambda_{C}$ is a measure of the size of the energy containing islands. The autocorrelation function is illustrated in Fig. 19(b). In the same figure $\langle\delta\rangle,\langle\ell\rangle$ are reported as vertical lines for comparison. The dissipation length, at which the turbu-

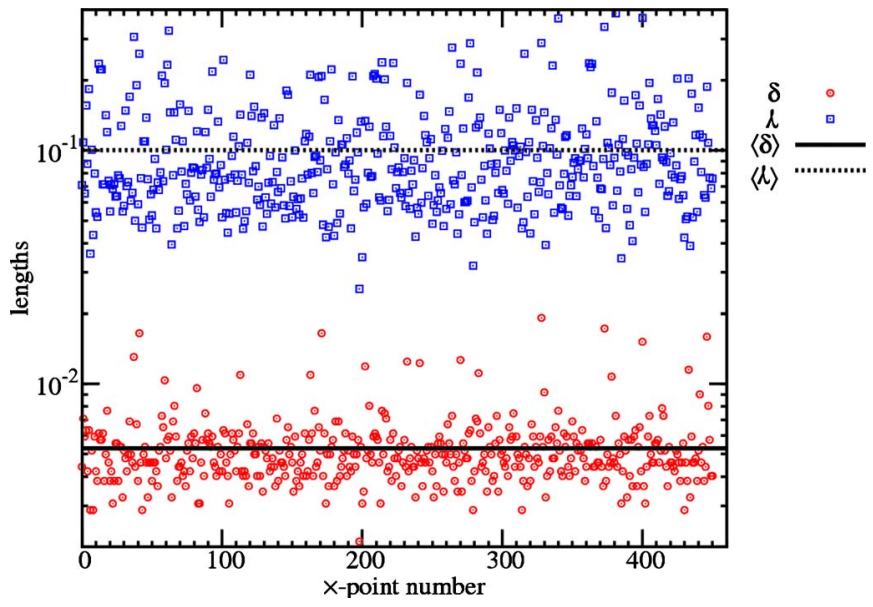

FIG. 17. (Color online) The values of $\delta$ (red circles) and $\ell$ (blue squares) for all the X-points. The solid and dashed lines represent the average values of $\delta$ and $\ell$, respectively.

lence is critically damped, is defined as $\lambda_{\text {diss }}=R_{\mu}^{-1 / 2}\left\langle j^{2}\right\rangle^{-1 / 4}$, while the Taylor microscale, a measure of mean-square gradients, is

$$
\lambda_{T}=\sqrt{\frac{\left\langle|\boldsymbol{b}|^{2}\right\rangle}{\left\langle j^{2}\right\rangle}} .
$$

In analogy to Eq. (18), we computed the second order structure functions as

$$
S(\mathbf{r})=\frac{\left\langle|\boldsymbol{b}(\boldsymbol{x}+\mathbf{r})-\boldsymbol{b}(\boldsymbol{x})|^{2}\right\rangle}{\left\langle b^{2}\right\rangle} .
$$

In Fig. 19(c) this function is shown. It appears that the average elongation $\ell$ is strongly related to the correlation length, where the structure function flattens, or, analogously, where $C(r)$ becomes zero. In all our simulations, as reported in Table II, we found that the values of diffusion layer thickness $\delta$ are distributed in the range between the Taylor scale and the dissipation scale, while the length $\ell$, though broadly scat-

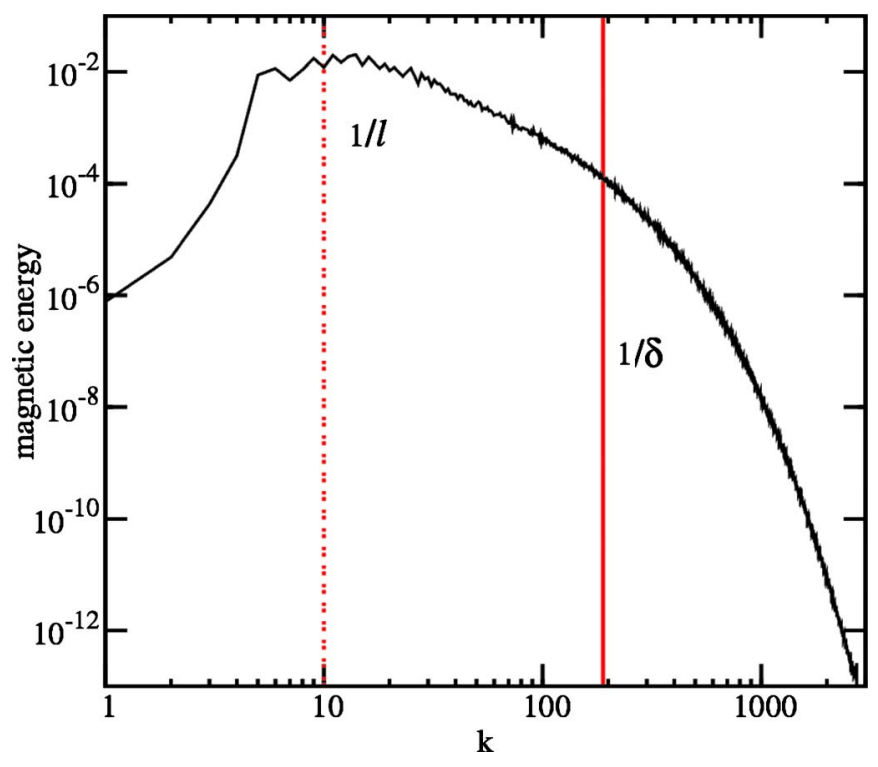

FIG. 18. (Color online) Magnetic spectrum together with $k_{\delta}$ and $k_{\ell}$. 


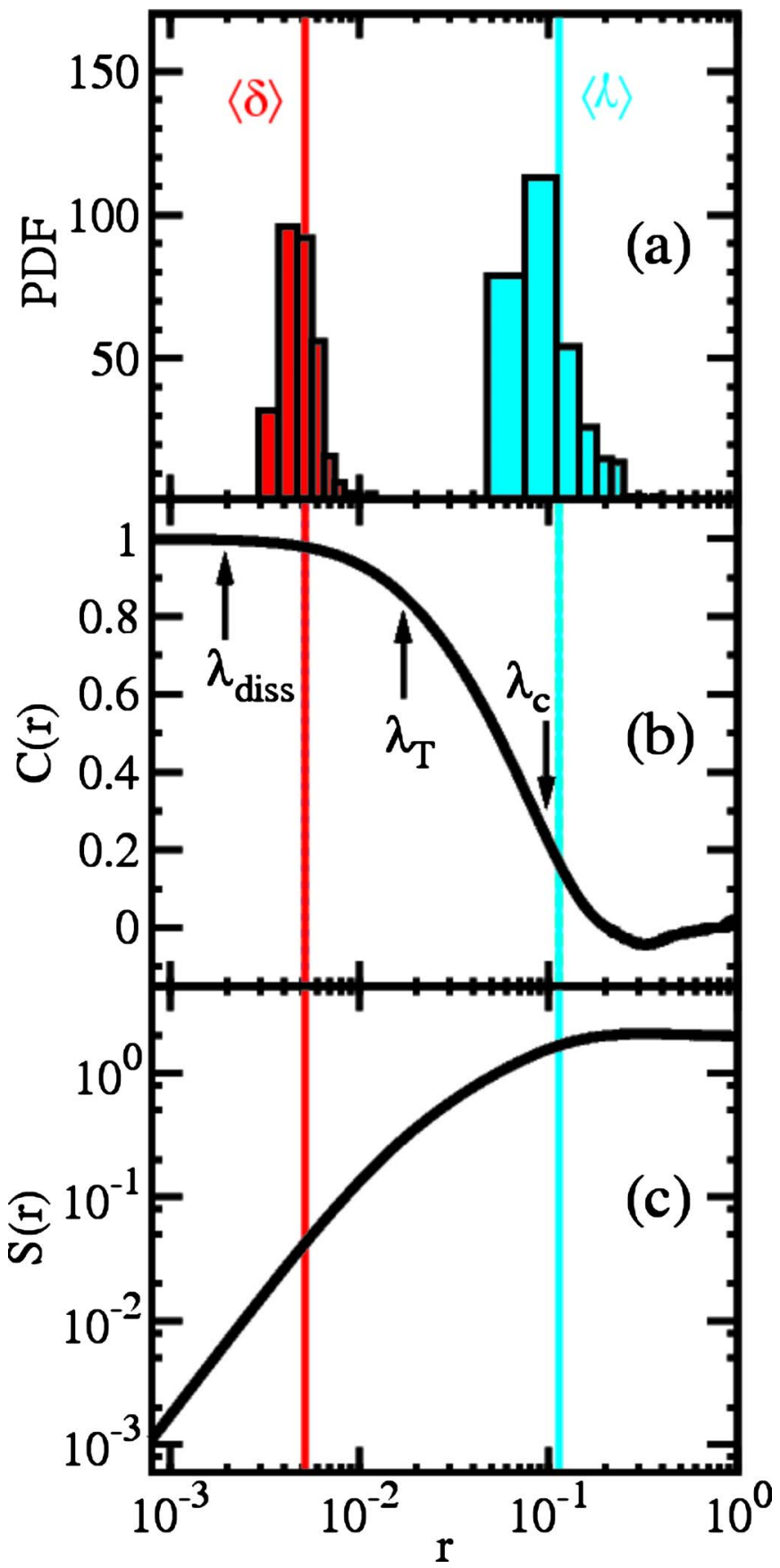

FIG. 19. (Color online) Top (a) histograms of thicknesses ( $\delta$, red bars) and elongations ( $\ell$, azure bars). In the middle panel (b) the magnetic field autocorrelation function (solid black line) is represented. Bottom plot (c) the structure function, defined by Eq. (20), is reported. The arrows (left to right) represent, respectively, dissipation scale $\lambda_{\text {diss }}$, Taylor microscale $\lambda_{T}$, and correlation length $\lambda_{C}$. Vertical lines are average values $\langle\delta\rangle$ (red) and $\langle\ell\rangle$ (azure). tered, scales with $\lambda_{C}$ (cf. Fig. 19). The main features of this ensemble of reconnecting events, including the key length scales, are evidently controlled by the statistical properties of turbulence, setting the range of values of length and thickness of the diffusion regions according to the correlation length and the dissipation scale. Note that a correlation between diffusion width and dissipation was discussed experimentally in Ref. 9.

\section{STEADY STATE SWEET-PARKER SCENARIO}

The turbulent reconnection activity identified above takes place in an environment in which the symmetric local conditions envisioned in standard laminar models are unlikely. It is therefore appropriate to employ the extension of the standard picture to asymmetric configurations. The Sweet-Parker-type analysis for asymmetric antiparallel reconnection has been studied in an earlier work by Cassak and Shay. ${ }^{54}$ In particular this analysis allows the reconnecting magnetic field strengths and plasma densities to be different on opposite sides of the dissipation region. Here we will summarize some of their main results. Asymmetric reconnection has also received recent attention in observations $^{61}$ and kinetic simulations. ${ }^{62}$

Using only conservation laws (mass continuity and Bernoulli theorem), without imposing any dissipation mechanism, it is possible to estimate the electric field in the diffusion region. ${ }^{54}$ In quasisteady state conditions the expected electric field at the X-point, i.e., the expected value of the reconnection rate, should scale as

$$
E_{\mathrm{cont}} \sim \frac{\left(b_{1} b_{2}\right)^{3 / 2}}{b_{1}+b_{2}} 2 \sqrt{\frac{\lambda_{\min }}{\lambda_{\max }}} \sim \frac{\left(b_{1} b_{2}\right)^{3 / 2}}{b_{1}+b_{2}} \frac{\delta}{\ell},
$$

where we specialize to the present notation and to the incompressible case.

Here we want to assess the accuracy of Eq. (21) for the computed turbulent reconnection sites. To do this we need to compute the upstream magnetic fields $b_{1,2}$. We used three different criteria to estimate $b_{1}$ and $b_{2}$. The results are illustrated in Fig. 20. The easiest way to estimate the upstream magnetic field [see Fig. 13] is to choose

$$
b_{1,2}=b_{t}\left(\delta_{1,2}\right) \text {, }
$$

which gives a reasonable estimation of the magnetic field near the diffusion region because the magnetic field here is very smooth and with a strong gradient. On the other hand, the values of $b_{1,2}$ might be underestimated in this way be-

\begin{tabular}{|c|c|c|c|c|c|}
\hline & $\lambda_{c}\left(\times 10^{-1}\right)$ & $\lambda_{T}\left(\times 10^{-2}\right)$ & $\lambda_{\text {diss }}\left(\times 10^{-3}\right)$ & $\langle\ell\rangle\left(\times 10^{-1}\right)$ & $\langle\delta\rangle\left(\times 10^{-2}\right)$ \\
\hline Run 1 & 2.27 & 4.80 & 5.10 & 2.80 & 1.44 \\
\hline Run 2 & 0.97 & 2.10 & 2.90 & 1.26 & 0.77 \\
\hline Run 3 & 2.36 & 4.40 & 4.10 & 2.32 & 1.20 \\
\hline Run 4 & 0.97 & 1.71 & 1.85 & 1.00 & 0.53 \\
\hline Run 5 & 1.18 & 2.15 & 2.00 & 1.13 & 0.62 \\
\hline Run 6 & 0.95 & 1.28 & 1.11 & 0.90 & 0.36 \\
\hline
\end{tabular}

TABLE II. Table of characteristic lengths. See text for more details. 


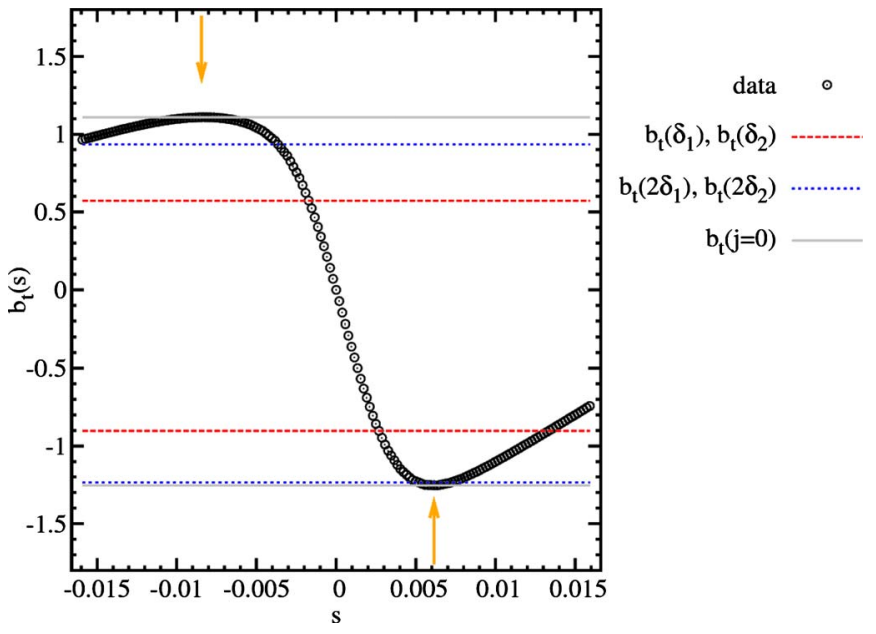

FIG. 20. (Color online) Tangential magnetic field upstream of a particular $\mathrm{X}$-point (black open circles) with different estimates for the upstream magnetic field (horizontal lines): $b_{\text {up }}$ evaluated using Eq. (22) (red dashed-lines), Eq. (23) (blue dotted-lines), and Eq. (24) (gray full-lines). The arrows indicate the points in which $j(s)=0$.

cause the magnetic field is too close to the neutral point, where $\boldsymbol{b}_{\times}=0$.

Another possible choice is

$$
b_{1,2}=b_{t}\left(2 \delta_{1,2}\right) \text {. }
$$

This approximation can also lead to some problems: it can be too far from the diffusion region. In some cases the magnetic field might decrease rapidly beyond the peak value, so that $b_{t}$ becomes very close to zero, giving a null reconnection rate. A more sophisticated way to derive a value of $b_{\text {up }}$ is to look at the current $j(s)$. As it can be seen from the examples in Fig. 13, the current density crosses the value zero on both the right and the left sides of the X-point. As mentioned above, this implies that the tangential magnetic field has maxima at these two points. Therefore, a third possible way to estimate the upstream magnetic field is

$$
\begin{aligned}
& b_{1,2}=b_{t}\left(s_{1,2}\right), \quad \text { where } \\
& j\left(s_{1,2}\right)=0 .
\end{aligned}
$$

In Fig. 20 we illustrate this algorithm, using Eqs. (22)-(24).

Using the above estimates for the two values of upstream magnetic field we can test the asymmetric conservation law to see whether it applies to this turbulent system. Figure 21 compares the reconnection rate $E_{\times}$from the simulations and the steady state estimates $E_{\text {cont }}$ from Eq. (21), for all the three techniques mentioned above. The best fit is obtained for the technique described by Eq. (24) [Fig. 21(c)]. [The fit errors are $\simeq 6.7 \%$ for the approximation given by Eq. (22), $\simeq 4.7 \%$ for Eq. (23), and $\simeq 4.5 \%$ for Eq. (24).] The good agreement with Eq. (21) indicates that the reconnection can be thought of as occurring in the quasisteady state. These results come only from conservation laws. In order to apply a resistive model that quantifies the reconnection rate, the dissipation mechanism should be described in detail.

In the incompressible case and in our notation, the Cassak-Shay ${ }^{54}$ asymmetric reconnection rate is given by
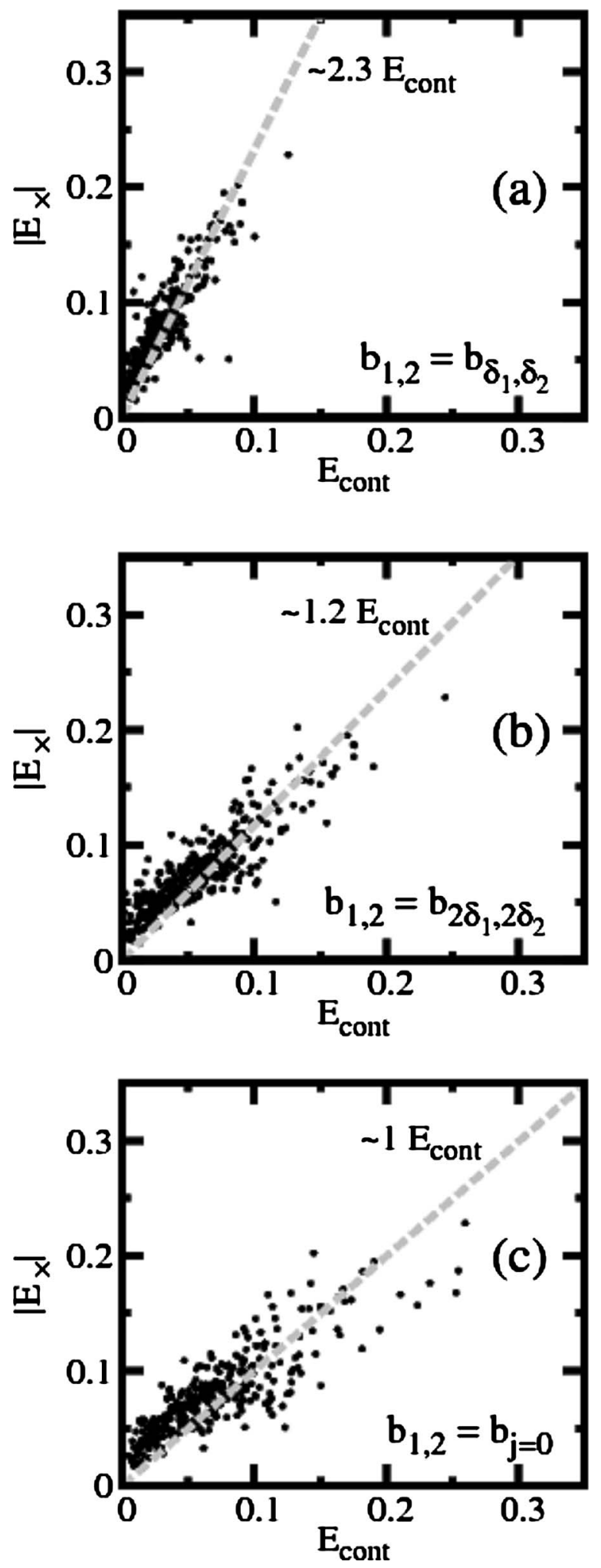

FIG. 21. Scatter plots of the electric field $E_{\times}$(in the fast reconnecting sites) vs the steady state function $E_{\text {cont }}$ (in absolute value) defined in Eq. (21). We computed $E_{\text {cont }}$ by using three different estimations of $b_{1,2}$, namely, by using Eq. (22) (a), Eq. (23) (b), and Eq. (24) (c).

$$
E_{\times}^{t h .} \simeq \sqrt{\frac{b_{1}^{3 / 2} b_{2}^{3 / 2}}{R_{\mu} \ell}} .
$$

Note that the present definition of $\delta$ and $\ell$ differ from those in Ref. 54 by a factor of 2 . However the intent of the scaling 


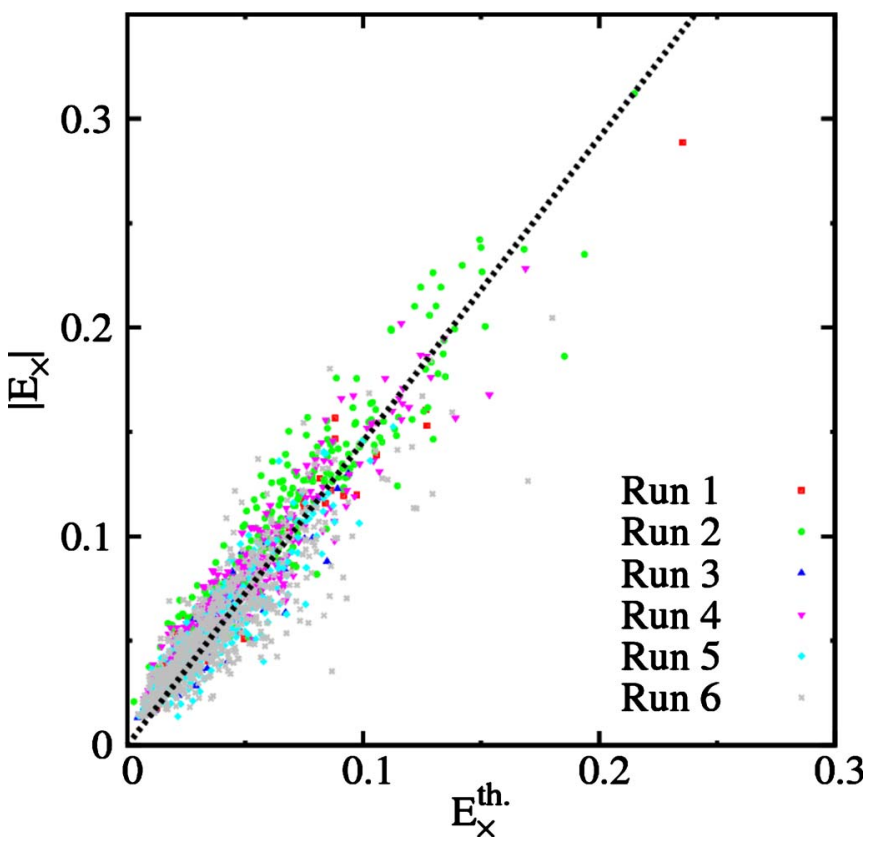

FIG. 22. (Color online) Computed reconnection rates vs expectation from Eq. (25) (Ref. 54). The good agreement indicates that the system is reconnecting in an asymmetric Sweet-Parker scenario.

relation Eq. (25) is insensitive to such factors. Here we would like to examine whether the observed ensemble of turbulent reconnection events scales as asymmetric "SweetParker" in this sense, with resistivity causing the dissipation. To acquire a broader picture of the scaling, we evaluated Eq. (25) using several runs (listed in Fig. 22). Figure 22 shows that in all the simulations the reconnection rates are consistent with the prediction given by Eq. (25). In this scenario turbulence plays a crucial role, determining locally the parameters that control the Sweet-Parker reconnection rate, namely, the lengths and local magnetic field strengths. Apparently, reconnection is an integral part of MHD turbulence cascade process.

\section{CONCLUSIONS}

The nonlinear dynamics of magnetic reconnection in turbulence has been investigated through direct numerical simulations of decaying 2D MHD. In the high resolution simulations considered here, many reconnection events are seen, involving simultaneously many magnetic islands of various size. The reconnection is spontaneous but locally driven by the fields and boundary conditions provided by the turbulence. Matching classical turbulence analysis with the Sweet-Parker theory, the statistical features of these multiscale reconnection events have been identified. Because of the complex magnetic topology, turbulence leads to different kinds of reconnecting patches.

The turbulent cascade produces a distribution of reconnecting islands. Computing the electric field at the X-points, we see that turbulence produces a broad range of reconnection rates, with values in excess of $0.1-0.3$ in dimensionless global Alfvén units. In addition, the strongest reconnection rates vary in proportion to $\ell / \delta$, the aspect ratio of the recon- nection sites. This scaling appears to differ greatly from classical laminar theories, ${ }^{17}$ but taking into account the nearby magnetic field produced by the turbulence, a form of generalized Sweet-Parker scaling ${ }^{54}$ is restored. These results explain how rapid reconnection occurs in MHD turbulence in association with the most intermittent non-Gaussian current structures, and also how turbulence generates a very large number of reconnection sites that have very small rates. Reconnection, like other transport processes, is greatly affected by turbulence ${ }^{63}$ and reconnection rates, like other turbulence parameters, have a broad distribution of values.

In contrast with laminar reconnection models that provide a single predicted reconnection rate for the system, turbulent resistive MHD gives rise to a broad range of reconnection rates that depend on local turbulence parameters. Many potential reconnection sites are present, but only a few are selected by the turbulence, at a given time, to display robust reconnection electric fields. In this way, the present problem differs greatly from studies of reconnection that assume that it occurs in isolation or as a spontaneous process. In those cases, the total electric field is due to reconnection, and in steady state, the electric field far from the diffusion region takes on the value of the reconnection electric field. Here the electric field at points removed from the X-points are typically larger than the reconnection rates. It seems to be appropriate therefore to view reconnection in turbulence as driven by the convective electric fields in the turbulent medium. It is this driving by the turbulent electric field that is responsible for the local flux pile-up that drives the reconnection discussed here. Flux pile-up would normally be viewed as occurring when the rate of approach of the islands is greater than the rate that can be supported by the reconnection process. This can cause buildup of repulsive forces and eventual bouncing. This will be an interesting feature to study in the turbulence context but such a time dependent analysis is beyond the scope of the present work.

We have seen that reconnection becomes an integral part of turbulence, as suggested previously. ${ }^{21}$ In fact, results of the present type may shed light on possible scalings as Reynolds numbers are increased, even though direct computational scalings remain greatly challenging. In particular, we expect that the distribution of reconnection rates can be related to the issue of maintaining finite energy dissipation in the infinite Reynolds numbers. A detailed examination of this connection remains for future study.

This new perspective on reconnection may be highly relevant to space and astrophysical applications such as the turbulent magnetosheath, ${ }^{8,9}$ the solar wind, ${ }^{7}$ and the solar corona. ${ }^{4}$ On the basis of the current results, we would expect to find in the turbulent corona and solar wind a broad distribution of size of interacting islands, with a concomitantly broad distribution of reconnection rates. The rates can, in principle, be determined statistically in terms of measurable correlation, Taylor and dissipation scales. In future studies it may be useful to study additional signatures such as characteristic reconnection flows. ${ }^{7}$ Furthermore a useful extension will be to employ models that are suited to low collisionality plasmas, where for example anomalous resistivity, Hall MHD, or other kinetic effects may be important. 


\section{ACKNOWLEDGMENTS}

Research supported by NSF grants (Solar Terrestrial Program) ATM-0539995, (SHINE) ATM-0752135, PHY0902479, and NASA grants NNG-06GD47G, NNZX07AR48G, NNX-08AM48G, (MMS) NNX-08AT76G, and (Heliophysics Theory) NNX-08AI47G. P.D. is a member of Carrera Investigador Cientifico of CONICET.

\section{APPENDIX: THE CELLULAR AUTOMATA ALGORITHM}

To investigate the coherent structures of the magnetic field, we use a cellular automata algorithm (CA) to analyze the magnetic potential function $a(x, y)$. The basic idea of the CA is to place some "seeds" in each O-point (maximum and minimum) of $a$, and then diffuse the seeds to all nearby points until reaching the threshold (set by some value or rule). The whole process comprises three steps.

First, identify all the critical points, including maximum, minimum, and saddle points. The square Hessian matrix of $a$ is $H_{i, j}^{a}(\boldsymbol{x})=\partial^{2} a / \partial x_{i} \partial x_{j}$. At each neutral point, where $\boldsymbol{\nabla} a=0$, we compute the eigenvalues of $H_{i, j}^{a}$. If both eigenvalues are positive (negative), the point is a local minimum (maximum) of $a$ (an O-point). If the eigenvalues are of mixed sign, it is a saddle point (an X-point). For periodic boundary, there will be equal amount of O-points and X-points.

Second, link every O-points to the closest X-point. Every maximum (minimum) point is connected to nearby maximum (minimum) points though X-points. To find the closest $\mathrm{X}$-point for each O-point, a simple CA algorithm is used, which will be explained below, taking a maximum as example.

Each position at the grid point is associated with a certain state, which is specified by a number $M_{i, j}$ where $i, j$ stand for the row and column of the grid point. $M_{i, j}$ can be $-1,0$ or 1 in this case. Initially, $M_{i, j}$ is set to be 1 for the four grid points which have that maximum inside the formed cell (red circles in Fig. 23), -1 for all the grid points which have an X-point inside the formed cell (black circles in Fig. 23), and 0 for all other grid points. A threshold value $A$ is set to be a very small negative integer, say $A=-10^{10}$. The evolution of $M_{i, j}$ from one step to the next follows the following rules.

If $M_{i, j}=1$, we check the value of $M_{i^{\prime}, j^{\prime}}$ for all nearby grid points $\left(i^{\prime}, j^{\prime}\right)$, where $i^{\prime}$ can be $i-1, i$ or $i+1$ and $j^{\prime}$ can be $j-1, j$ or $j+1$ :

(a) If $M_{i^{\prime}, j^{\prime}}=0$ and $a_{i^{\prime}, j^{\prime}}>A$, then $M_{i^{\prime}, j^{\prime}}$ is set to be 1 .

(b) If $M_{i^{\prime}, j^{\prime}}=-1$ and $a_{i^{\prime}, j^{\prime}}>A, M_{i, j}$ is reset to be the initial value for all grid points and the threshold value $A$ is set to be $a_{i^{\prime}, j^{\prime}}+\epsilon$, where $\epsilon$ is a very small positive constant.

Following these rules, a steady state can be achieved when $M_{i, j}$ does not change again and the final threshold value is marked to be $A_{n}$ (for the $n$th O-point). Thus the closest $\mathrm{X}$-point is defined in $a$ value, but not in distance, so that when the $a$ value at the X-point, $a(X)$ is used as threshold value, the region identified by the CA will not extend to any other X-point.

Finally, after the linked X-point and threshold values are found for every O-point, a similar CA is used in the whole

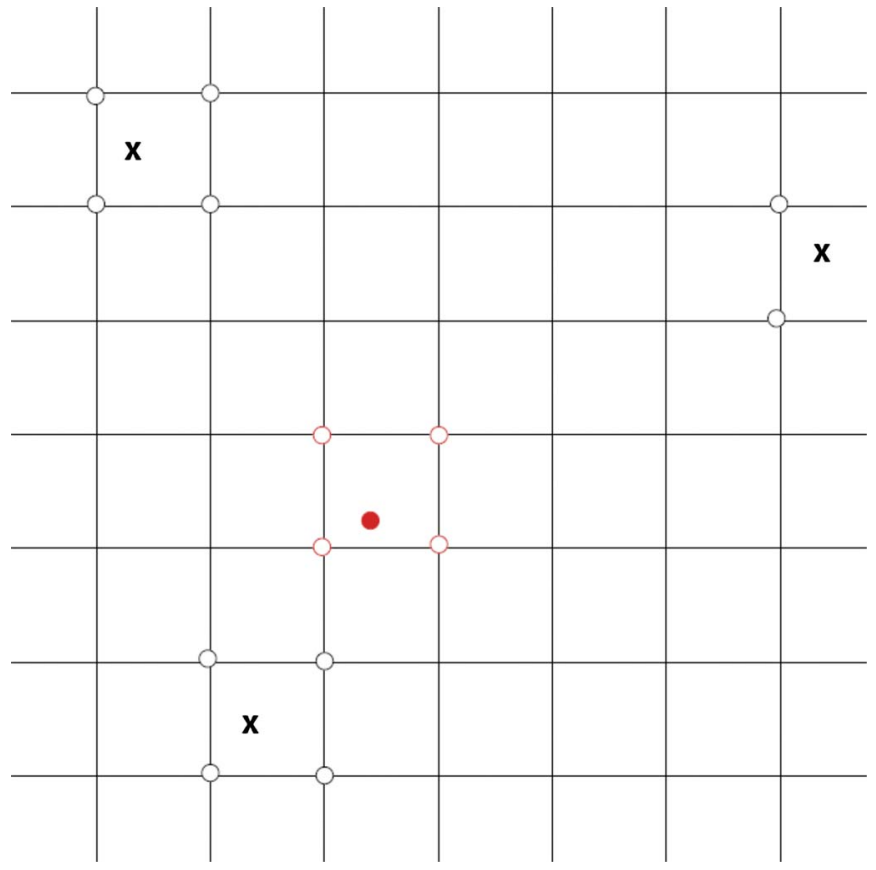

FIG. 23. (Color online) A sample grid with O-points (red solid point) and $\mathrm{X}$-points ("x").

area. Different seeds are placed on every O-points and diffused until reaching the corresponding threshold value $A_{n}$.

In this way, spatial patches (magnetic islands) can be identified. For the $n$th island, it will have an O-point $\mathbf{O}_{n}$ at the center and is bounded by field line with $a=A_{n}$. In Fig. 12, we show contour lines of $a$ together with the all magnetic islands detected by the cellular automata algorithm.

${ }^{1}$ B. U. O. Sonnerup, J. Plasma Phys. 4, 161 (1970).

${ }^{2}$ V. M. Vasyliunas, Rev. Geophys. Space Phys. 13, 303, doi:10.1029/ RG013i001p00303 (1975).

${ }^{3}$ H. K. Moffatt, Magnetic Field Generation in Electrically Conducting Fluids (Cambridge University Press, Cambridge, 1978).

${ }^{4}$ E. N. Parker, Astrophys. J. 264, 642 (1983).

${ }^{5}$ G. Paschmann, Geophys. Res. Lett. 35, L19109, doi:10.1029/ 2008GL035297 (2008).

${ }^{6}$ B. U. O. Sonnerup, G. Paschmann, I. Papamastorakis, N. Sckopke, G. Haerendel, S. J. Bame, J. R. Asbridge, J. T. Gosling, and C. T. Russel, J. Geophys. Res. 86, 10049, doi:10.1029/JA086iA12p10049 (1981).

${ }^{7}$ J. T. Gosling and A. Szabo, J. Geophys. Res. 113, A10103, doi:10.1029/ 2008JA013473 (2008).

${ }^{8}$ A. Retinò, D. Sundkvist, A. Vaivads, F. Mozer, M. André, and C. J. Owen, Nat. Phys. 3, 236 (2007).

${ }^{9}$ D. Sundkvist, A. Retinò, A. Vaivads, and S. D. Bale, Phys. Rev. Lett. 99, 025004 (2007).

${ }^{10}$ B. B. Kadomtsev, Sov. J. Plasma Phys. 1, 389 (1975).

${ }^{11}$ Y. Yamada, Phys. Plasmas 14, 058102 (2007).

${ }^{12}$ H. Ji, M. Yamada, S. Hsu, and R. Kulsrud, Phys. Rev. Lett. 80, 3256 (1998).

${ }^{13}$ M. R. Brown, C. D. Cothran, and J. Fung, Phys. Plasmas 13, 056503 (2006).

${ }^{14}$ J. B. Taylor, Rev. Mod. Phys. 58, 741 (1986).

${ }^{15}$ J. W. Dungey, Cosmic Electrodynamics (Cambridge University Press, New York, 1958).

${ }^{16}$ K. Schindler, M. Hesse, and J. Birn, J. Geophys. Res. 93, 5547, doi:10.1029/JA093iA06p05547 (1988).

${ }^{17}$ P. A. Sweet, Electromagnetic Phenomena in Cosmical Physics (Cambridge University Press, New York, 1958).

${ }^{18}$ E. N. Parker, J. Geophys. Res. 62, 509, doi:10.1029/JZ062i004p00509 (1957).

${ }^{19}$ T. Sato, T. Hayashi, and T. Tamao, Phys. Rev. Lett. 41, 1548 (1978). 
${ }^{20}$ J. Birn, J. F. Drake, M. A. Shay, B. N. Rogers, R. E. Denton, M. Hesse, M. Kuznetsova, Z. W. Ma, A. Bhattacharjee, A. Otto, and P. L. Pritchett, J. Geophys. Res. 106, 3715, doi:10.1029/1999JA900449 (2001).

${ }^{21}$ W. H. Matthaeus and D. Montgomery, Ann. N.Y. Acad. Sci. 357, 203 (1980).

${ }^{22}$ W. H. Matthaeus and S. L. Lamkin, Phys. Fluids 29, 2513 (1986).

${ }^{23}$ H. R. Strauss, Astrophys. J. 326, 412 (1988).

${ }^{24}$ V. Carbone, P. Veltri, and A. Mageney, Phys. Fluids A 2, 1487 (1990).

${ }^{25}$ P. Dmitruk and W. H. Matthaeus, Phys. Plasmas 13, 042307 (2006).

${ }^{26}$ F. Malara, P. Veltri, and V. Carbone, Phys. Fluids B 4, 3070 (1992).

${ }^{27}$ A. Lazarian and E. Vishniac, Astrophys. J. 517, 700 (1999).

${ }^{28}$ G. Lapenta, Phys. Rev. Lett. 100, 235001 (2008).

${ }^{29}$ S. Servidio, W. H. Matthaeus, M. A. Shay, P. A. Cassak, and P. Dmitruk, Phys. Rev. Lett. 102, 115003 (2009).

${ }^{30}$ H. Furth, J. Kileen, and M. N. Rosenbluth, Phys. Fluids 6, 459 (1963).

${ }^{31}$ P. H. Rutherford, Phys. Fluids 16, 1903 (1973).

${ }^{32}$ H. Petschek, Physics of Solar Flares (NASA SP-50, Washington, D.C., 1964) p. 425

${ }^{33}$ A. Ishizawa, R. Horiuchi, and H. Ohtani, Phys. Plasmas 11, 3579 (2004).

${ }^{34}$ J. Huba and L. I. Rudakov, Phys. Rev. Lett. 93, 175003 (2004).

${ }^{35}$ W. H. Matthaeus and D. Montgomery, J. Plasma Phys. 25, 11 (1981).

${ }^{36}$ W. H. Matthaeus and S. L. Lamkin, Phys. Fluids 28, 303 (1985).

${ }^{37}$ D. Smith, S. Ghosh, P. Dmitruk, and W. H. Matthaeus, Geophys. Res. Lett. 31, L02805, doi:10.1029/2003GL018689 (2004).

${ }^{38}$ E. Kim and P. H. Diamond, Phys. Lett. A 291, 407 (2001)

${ }^{39}$ G. Kowal, A. Lazarian, E. T. Vishniac, and K. Otmianowska-Mazur, Astrophys. J. 700, 63 (2009).

${ }^{40}$ N. F. Loureiro, D. A. Uzdensky, A. A. Schekochihin, S. C. Cowley, and T. A. Yousef, Mon. Not. R. Astron. Soc. 399, L146 (2009).

${ }^{41}$ W. Daughton, V. Roytershteyn, B. J. Albright, H. Karimabadi, L. Yin, and J. K. Bowers, Phys. Rev. Lett. 103, 065004 (2009).

${ }^{42}$ P. A. Cassak, M. A. Shay, and J. F. Drake, Phys. Plasmas 16, 120702 (2009).

${ }^{43}$ A. Bhattacharjee, Y.-M. Whang, H. Yang, and B. Rodgers, Phys. Plasmas 16, 112102 (2009).
${ }^{44}$ D. Biskamp, Nonlinear Magnetohydrodynamics (Cambridge University Press, Cambridge, 1993).

${ }^{45}$ S. Ghosh, M. Hossain, and W. H. Matthaeus, Comput. Phys. Commun. 74, 18 (1993).

${ }^{46}$ S. Servidio, W. H. Matthaeus, and P. Dmitruk, Phys. Rev. Lett. 100, 095005 (2008).

${ }^{47}$ A. Greco, P. Chuychai, W. H. Matthaeus, S. Servidio, and P. Dmitruk, Geophys. Res. Lett. 35, L19111, doi:10.1029/2008GL035454 (2008).

${ }^{48}$ S. Rana, Surface Topological Data Structures: An Introduction for Geographical Information Science (Wiley, Chichester, 2004).

${ }^{49}$ L. Florack and A. Kuijper, J. Math. Imaging Vision 12, 65 (2000).

${ }^{50}$ L. J. Milano, W. H. Matthaeus, B. Breech, and C. W. Smith, Phys. Rev. E 65, 026310 (2002).

${ }^{51}$ B. Breech, W. H. Matthaeus, L. J. Milano, and C. W. Smith, J. Geophys. Res. 108, 1153, doi:10.1029/2002JA009529 (2003).

${ }^{52}$ R. H. Kraichnan and R. Panda, Phys. Fluids 31, 2395 (1988).

${ }^{53}$ M. Wan, S. Oughton, S. Servidio, and W. H. Matthaeus, Phys. Plasmas 16, 080703 (2009).

${ }^{54}$ P. A. Cassak and M. A. Shay, Phys. Plasmas 14, 102114 (2007).

${ }^{55}$ J. C. Dorelli and J. Birn, J. Geophys. Res. 108, 1133, doi:10.1029/ 2001JA009180 (2003).

${ }^{56}$ J. D. Craig and P. G. Watson, Astrophys. J. 516, 924 (1999).

${ }^{57}$ T. J. Bogdan, Phys. Fluids 27, 994 (1984).

${ }^{58}$ E. G. Zweibel and J. E. Rhoads, Astrophys. J. 440, 407 (1995).

${ }^{59}$ T. P. Intrator, X. Sun, G. Lapenta, L. Dorf, and I. Furno, Nat. Phys. 5, 521 (2009).

${ }^{60}$ W. H. Press, B. P. Flannery, and S. A. Teukolsky, Numerical Recipes (Cambridge University Press, New York, 1986), pp. 387-436.

${ }^{61}$ F. S. Mozer and P. L. Pritchett, Geophys. Res. Lett. 36, L07102, doi:10.1029/2009GL037463 (2009).

${ }^{62}$ P. L. Pritchett and F. S. Mozer, J. Geophys. Res. 114, A11210, doi:10.1029/2009JA014343 (2009).

${ }^{63}$ S. A. Orszag, in Fluid Dynamics: Les Houches Summer School, 1973, edited by R. Balian and J.-L. Peube (Gordon and Breach, New York, 1977), p. 235. 